\title{
CNAHap: a germline haplotyping method using tumor allele-specific copy number alteration
}

\author{
Bowen Tan ${ }^{1, \dagger}$, Lingxi Chen ${ }^{1, \dagger}$, Wenlong Jia ${ }^{1}$, Yanfei Wang ${ }^{1}$, Hechen $\mathrm{Li}^{1,2}$, and Shuai Cheng $\mathbf{L i}^{1, *}$ \\ ${ }^{1}$ Department of Computer Science, City University of Hong Kong, Hong Kong, China \\ ${ }^{2}$ School of Computational Science and Engineering, Georgia Institute of Technology, Atlanta GA 30332, USA \\ *Corresponding: shuaicli@cityu.edu.hk \\ ${ }^{\dagger}$ These authors contributed equally to this work.
}

\begin{abstract}
Haplotype phasing is indispensable to study human genetics. 44 The pervasiveness of large copy number variant segments in 45 solid tumors brings possibilities to resolve long germline phasing blocks utilizing allele imbalance in tumor data. Although there exist such studies, none of them provide easy-use software based on availability and usability. Herein, we present a novel tool, CNAHap, to determine the allele-specific copy number in tumor and then phase germline variants according to the imbalanced alleles in tumor genomes. We also provide interactive web interfaces to visualize the copy number and phase land- 52 scape from CNAHap. On in silico datasets, CNAHap demon- 53 strates higher allele-specific copy number calling accuracy than the benchmark tool and generates long phasing blocks. As a case study on Hepatocellular carcinoma, CNAHap successfully generates huge phase blocks with the averages of N50 and N90 as $25 \mathrm{M}$ and $7 \mathrm{M}$, respectively, and finds the Olfactory receptor family is recurrent amplified. Our results illustrate the efficacy of CNAHap in determining tumor allele-specific copy numbers ${ }^{59}$ and their long germline haplotypes. CNAHap is available at 60 https://github.com/bowentan/CNAHap and the CNA- 61 Hap visualization web interfaces are hosted at bio . oviz . org. 62
\end{abstract}

lotypes employ two strategies (9). The first one utilizes the population database to phase while demonstrates the inability of handling rare and de novo variants (10). The latter strategy is to assemble the haplotype from the sequencing reads. Mainstream haplotype assembly tools catalog the genetic variants of the germline haplotype by incorporate the linkage information from high-throughput sequencing of normal tissue (11-16). Nevertheness, the length of the phased block, and the number of phased SNVs/InDels rely on the read linkages.

To further extend the phased block, some studies incorporate tumor data to unveil germline haplotypes. Large somatic copy number aberration (SCNA) blocks are prevalent (almost $90 \%$ ) in solid tumors (17). Equipped with tumor allele frequency, now scientists can phase over the large copy number aberration (CNA) blocks and are free from the read length and insert size of a sequencing protocol, promoting a higher phase rate than merely adopting normal data (18). HATS (19) is a population-based approach that adopts a hidden Markov model to construct germline haplotypes in copy number variation (CNV) gain regions. VAF phasing (18) forms germline haplotypes by distinguishing variant allele frequency (VAF) changes between paired tumor and normal tissues in areas of $\mathrm{CNV}$ gains. However, running these tools requires arduous user interventions as VAF phasing provides no open-source software and HATS necessitates a training process first.

In this work, we spotlight germline phasing with tumor CNA, and propose a novel user-friendly tool, CNAHap (https://github.com/bowentan/CNAHap, Figure 1), to phase SNVs/InDels as in normal cells by taking advantage of allele imbalance from paired tumor CNV blocks. CNAHap also calls the allele-specific copy number aberrations in tumor cells. In addition, to visualize the CNAHap output vividly, we developed three online interactive visualization applications (CNV: Circos View, CNV: Focal Cluster, and Phased: On Genes) hosted in Bio-Oviz bio.oviz.org (20) (Table 1). We validated the phasing efficacy of CNAHap in three in silico WGS data sets with 
different tumor purity rates, and CNAHap exhibits a higher ${ }_{136}$ allele-specific copy number calling accuracy than the bench- ${ }_{137}$ mark tool and generates long phasing blocks. Then we con- ${ }_{138}$ ducted a case study in a Hepatocellular carcinoma (HCC) ${ }_{139}$ cohort. CNAHap successfully generates huge phase blocks with the average N50 and N90 as 25M and 7M, respectively, 140 and finds the Olfactory receptor family is recurrent amplified. ${ }_{141}$

\section{Materials and methods}

To estimate germline haplotypes from normal and tumor samples, CNAHap consists of two components. The first is to estimate allele-specific copy numbers, i.e., the copy numbers of the two haplotypes of given segments in tumor cells. The second is to perform the SNV phasing on segments by the fact that SNVs along the same haplotype sharing a similar copy rate. When a CNV event occurs, the alleles with larger depths seem to be along one haplotype, and the alleles with smaller depth aligns with the other haplotype.

Target SNV extraction. Given the sequencing data of nor- ${ }^{154}$ mal and tumor cell samples from a cancer patient, CNAHap ${ }^{155}$ is designed to find two haplotypes of SNV loci which are supposed to be originated from the germline, hence they are contained extensively in all types of cells, such as tissue and germline cells. Therefore, a shared set of heterozygous SNVs as the target SNVs loci are extracted from normal and tu- 156 mor cell samples by selecting SNVs with the same identifiers ${ }^{157}$ including contig names, positions, reference alleles and al- 158 ternative alleles between normal and tumor cells. All subsequent analysis will be performed merely on these target SNVs.

Allele imbalance and copy number estimation. Before 160 estimating haplotypes, CNAHap first needs to estimate allele- 161 specific copy numbers of the given $\mathrm{CNV}$ segments. If a CNV 162 event occurs in a genomic region or a genomic segment, three possible outcomes will arise. The first is segments with imbalanced copies, because of different numbers of copies two haplotypes are duplicated. The second is balanced segments with the same number of copies. The third outcome is that ${ }_{163}$ one of the haplotypes disappears due to deletion and the other ${ }_{164}$ haplotype remains one copy or changes to multiple copies. ${ }_{165}$ As a result, $\mathrm{SNV}$ s from the first outcomes have the potential ${ }_{166}$ to contribute imbalance characteristics for the haplotype es- ${ }_{167}$ timation and hence are possible to be phased. The segments ${ }_{168}$ from the other two outcomes are either unable to provide significant evidence to separate the two haplotypes because of comparable allele depths or possess only homozygous SNVs. For our concern, therefore, SNVs in segments of the first outcome are the targets to be phased.

Parameters to be estimated. Assume there are $N$ CNV segments concerned. Here we aim to estimate the copy numbers ${ }^{171}$ of the major $H$ and the minor $h$ haplotypes in a tumor sam- 172 ple; denote them as $C_{H, i}$ and $C_{h, i}$ for segment $i, 1 \leq i \leq N$. 173 Since normal and tumor cells may coexist in the samples, 174 reads from normal and tumor cells may be mixed in sequenc- 175 ing data. There arises a parameter, tumor purity rate $\rho$, to be 176 concerned. The purity is the proportion of tumor cells in a mixed sample.

We can extract different features from the input datasets, and these features would constrain the parameters.

Constraints according to allele depths. From the tumor sequencing data, we can calculate for sequencing depth $D_{H, i}$ and $D_{h, i}$ for the major and minor haplotype $H$ and $h$ for each segment $i$, respectively. Moreover, we can estimate the amplification factor $D$; that is, the number of times a single copy of a haplotype is sequenced in the tumor dataset. $D_{H, i}$ and $D_{h, i}$ can be computed from the variant call format (VCF) file, as the average across the loci. We adopted K-means clustering to estimate $D$. We normalize the depth of a segment by the depth of the whole tumor data set to calculate the copy numbers initially. Then we choose the number of integers from the rounded minimum copy numbers as the the number of clusters for the K-means. Finally, we pick half of the average depth of the segments in the cluster with the minimum cluster centroid as $D$.

Now, we can constrain $D_{H, i}$ and $D_{h, i}$ as

$$
\begin{aligned}
& D_{H, i} \approx\left((1-\rho)+\rho C_{H, i}\right) D \\
& D_{h, i} \approx\left((1-\rho)+\rho C_{h, i}\right) D .
\end{aligned}
$$

Constraints according to segment depth. From the input, we can calculate the average depth $D_{i}$ for segment $i$, hence, we can constrain the parameters as

$$
D_{i} \approx\left(2(1-\rho)+\rho C_{H, i}+\rho C_{h, i}\right) D
$$

Constraints according to depth differences. The average difference $S_{i}$ can be computed from input between the two haplotypes for segment $i$, and it should be also comparable with the one calculated from $\rho, C_{H, i}$ and $C_{h, i}$, that is

$$
S_{i} \approx \rho\left(C_{H, i}-C_{h, i}\right) D
$$

Constraints according to allele imbalance. We define $\Lambda_{i}$ as the allele imbalance (AI) for segment $i$, which is a weighted average of the AI values at all the heterogeneous loci in the segment. Assume the segment $i$ harbours heterogeneous locus set $K_{i}$. Denote $\lambda_{k}$ and $w_{k}$ as the AI value and weight of locus $k$, respectively. Then we have

$$
\Lambda_{i}=\frac{\sum_{k \in K i} w_{k} \lambda_{k}}{\sum_{k \in K i} w_{k}}
$$

Below we specify how to obtain $\lambda_{k}$ and $w_{k}$. Denote the allele depths as $d_{k, r}$ and $d_{k, a}$ at the $k$-th locus for reference, and alternatives, respectively, then $\lambda_{k}=\frac{\left|d_{k, r}-d_{k, a}\right|}{\max \left\{d_{k, r}, d_{k, a}\right\}}$.

Under the assumption that the reference and alternative alleles will be sequenced by equal chance if the segment is balanced, i.e., $q_{k, r}=\operatorname{Pr}$ (reference is sequenced $)=0.5$, the allele depth of a variant should follow a binomial distribution (see Equation Eq. (5)). 

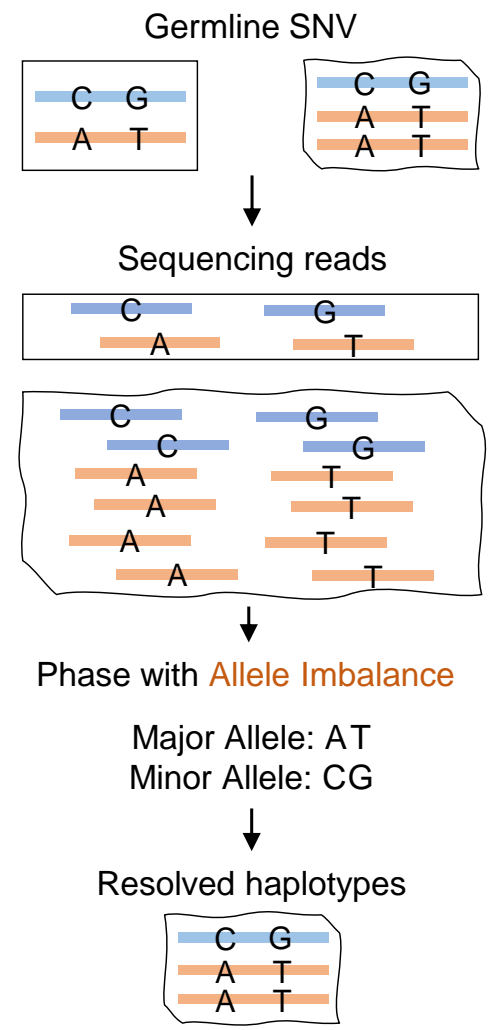

b

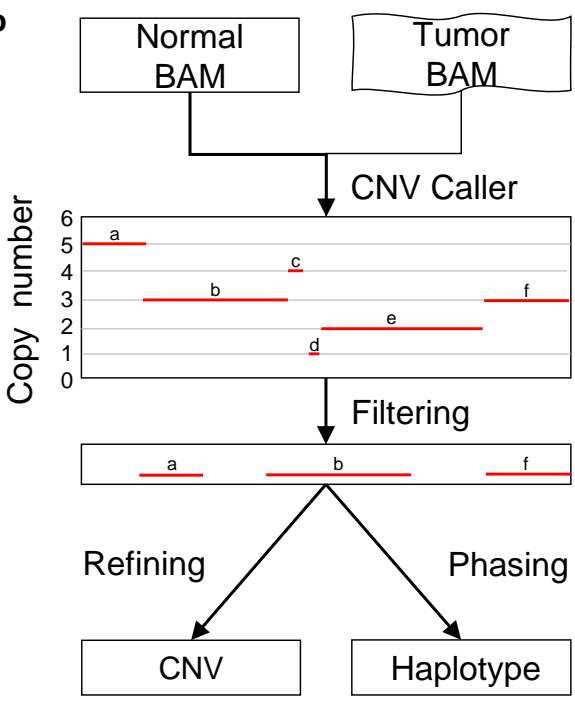

Filtering:

Filter out CNV segment which

- contains little SNVs "c" "d"

- not allele imbalanced "e"

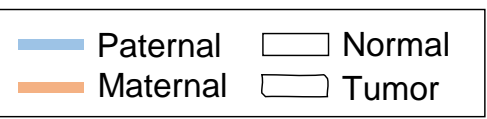

C

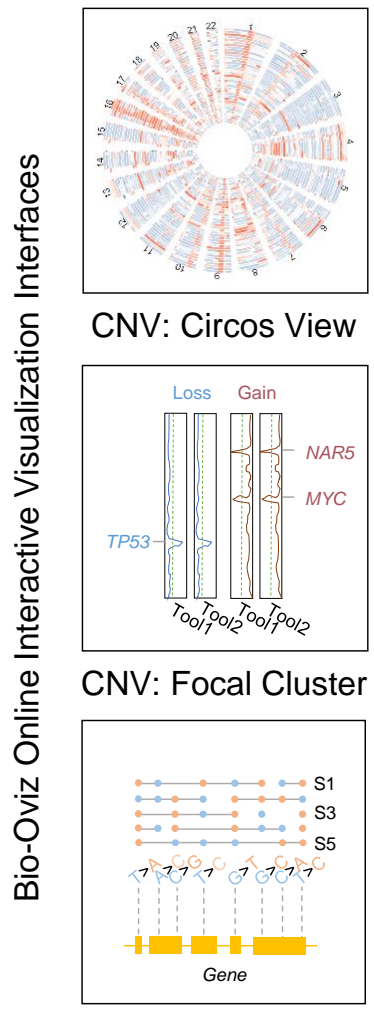

Phased: On Genes

Fig. 1. Overview of CNAHap. (a) The core principle of CNAHap to phase. (b) The workflow of CNAHap. (c) Three online interactive visualization interfaces hosted in bio. oviz. org Oviz-Bio (20).

$$
p_{k}=\operatorname{Pr}\left(d_{k, r}, d_{k, a}\right)=2\left(\begin{array}{c}
d_{k, r}+d_{k, a} \\
d_{k, r}
\end{array}\right) q_{k, r}^{d_{k, r}}\left(1-q_{k, r}\right)^{d_{k, a}}{ }^{192}
$$

Then we formulate the weight $w_{k}$ for the $k$-th variant as Equation Eq. (6). Phred quality scores $Q$ are defined as a property which is logarithmically related to the base-calling error probability $\mathrm{P}$

$$
w_{k}=-10 \log _{10} p_{k}
$$

Such a weight has a property that the more imbalanced the allele depths are, the larger the weight will be since the binomial coefficient in $p_{k}$ will be smaller. The AI $\Lambda_{i}$ values should be compatible from these calculated from the parameters; that is,

$$
\Lambda_{i} \approx \frac{\rho\left(C_{H, i}-C_{h, i}\right)}{(1-\rho)+\rho C_{H, i}} .
$$
Solving the parameters with integer programming. With the ${ }_{197}$ aforementioned constraints, we implement an integer pro- ${ }_{198}$ gramming (IP) to estimate $C_{H, i}$ and $C_{h, i}$ for each segment. ${ }_{199}$ We replace the approximations by error variables $\epsilon$, where we ${ }_{200}$ want to minimize the sum of errors.

$$
C_{H, i} \geq C_{h, i}, C_{H, i}, C_{h, i} \in \mathbb{I}
$$

where $\mathbb{I}$ is the integer set.

Combining the constraints Eq. (1), Eq. (2), Eq. (3), Eq. (7) and Eq. (8), we summarize the model in Eq. (9).

$$
\begin{array}{ll}
\min & \sum_{i=1}^{N}\left(\epsilon_{i, D}+\epsilon_{i, M}+\epsilon_{i, m}+\epsilon_{i, S}+\epsilon_{i, \Lambda}\right) \\
\text { s.t. } & \left|\left(2(1-\rho)+\rho C_{H, i}+\rho C_{h, i}\right) D-D_{i}\right| \leq \epsilon_{i, D} \\
& \left|\left((1-\rho)+\rho C_{H, i}\right) D-D_{H, i}\right| \leq \epsilon_{i, H} \\
& \left|\left((1-\rho)+\rho C_{h, i}\right) D-D_{h, i}\right| \leq \epsilon_{i, h} \\
& \left|\rho\left(C_{H, i}-C_{h, i}\right) D-S_{i}\right| \leq \epsilon_{i, S} \\
& \left|\frac{\rho\left(C_{H, i}-C_{h, i}\right)}{(1-\rho)+\rho C_{H, i}}-\Lambda_{i}\right| \leq \epsilon_{i, \Lambda} \\
& C_{H, i} \geq C_{h, i}, C_{H, i}, C_{h, i} \in \mathbb{I}
\end{array}
$$

Haplotype estimation. Having estimated the major and minor copy numbers of segments in pure tumor cells, CNAHap will proceed to perform phasing. With $C_{H, i}$ 's and $C_{h, i}$ 's, CNAHap will phase SNVs along the segments where $C_{H, i}>C_{h, i}$. Before phasing, the allele depths of SNVs in each segment will be updated to the allele depth in pure tumor cells. For segment $i$, we first calculate the fractions of major and minor depths $\left(f_{i, H}\right.$ and $\left.f_{i, h}\right)$ contributed by tumor cells using the purity $\rho$ and the major and minor copy numbers, $C_{H, i}$ and $C_{h, i}$ (see Equation Eq. (10)). 
205

$$
\begin{aligned}
f_{i, H} & =\frac{\rho C_{H, i}}{(1-\rho)+\rho C_{H, i}} \\
f_{i, h} & =\frac{\rho C_{h, i}}{(1-\rho)+\rho C_{h, i}}
\end{aligned}
$$

Then we update the allele depths for all SNVs in the segment, ${ }^{25}$ multiplying the observed allele depths by the corresponding ${ }^{252}$ fraction.

$$
\begin{aligned}
d_{k, H}^{\prime} & =d_{k, H} f_{i, H} \\
d_{k, h}^{\prime} & =d_{k, h} f_{i, h}
\end{aligned}
$$

Finally, we perform the phasing for the segment by compar- 259 ing the updated depths for all SNVs and treating the major 260 alleles of all SNVs as the variants from one haplotype and the ${ }_{26}$ minor alleles from the other. Therefore, the two haplotypes 262 $H_{i}$ and $h_{i}$ of a segment involving $n$ SNVs can be obtained as 263

$$
\begin{aligned}
H_{i} & =\left\{s_{k} \mid s_{k}=0 \text { if } d_{k, r}^{\prime}>d_{k, a}^{\prime}, \text { else } s_{k}=1, k=1 \ldots n\right\} \\
h_{i} & =\left\{s_{k} \mid s_{k}=0 \text { if } d_{k, r}^{\prime}<d_{k, a}^{\prime}, \text { else } s_{k}=1, k=1 \ldots n\right\}
\end{aligned}
$$

\section{Results}

Overview of CNAHap. Each individual obtains two copies 270 of chromosomes from parents separately. All genetic markers along the personal genome, such as single nucleotide variants (SNVs), small insertions and deletions (InDels), and short tandem repeats (STRs) should maintain the same across cells, ${ }_{275}$ except for somatic mutations occurring in tumor cells. Fur- ${ }_{276}$ thermore, the order and sequence of markers along local re- ${ }_{277}$ gions of chromosomes are the same as these inherited from ${ }_{278}$ parents. If copy number variations occur in some genome ${ }_{279}$ areas in tumor cells, these regions will gain extra copies. ${ }_{280}$ Hence, one or both haplotypes regarding these regions should ${ }_{281}$ be duplicated multiple times. Therefore, copies of two haplotypes in a genomic region in tumor cells may become imbal- 282 anced, and variants of markers in such a region may have im- 283 balanced sequencing depths, providing evidence for the orig- 284 inal haplotypes (Figure 1a). Here, we produced a computation tool, CNAHap, that adopts 286 the above mentioned principle as core to phase. The skeleton ${ }_{287}$ for CNAHap was illustrated in Figure 1b. First, with paired 288 tumor and normal BAM files, CNV caller Accurity (21) is 289 adopted to call the CNV blocks and tumor purity. CNAHap 290 then estimates allele-specific copy numbers for segments of 291 allele imbalance with an integer programming model and fil- 292 ters out those which contain little SNV locus or are allele bal- 293 anced. Then the phasing algorithm is then performed on each 294 filtered CNV segment along each chromosome. Third, CNA- 295 Hap outputs the resolved haplotype in VCF format, which 296 benefits subsequent analysis and interpretation. Finally, with 297 auxiliary annotation and downstream analysis scripts, the 298 output of CNAHap can be interactively visualized in CNV: 299 Circos View, CNV: Focal Cluster, and Phased: On Genes, sоo hosted on bio.oviz . org Bio-Oviz (20) (Figure 1c).
Evaluation of CNAHap on in silco data. To evaluate the sensitivity of CNAHap, we invested in silico mixtures of sequencing reads from the normal-tumor pair with increased proportions to simulate different tumor purity ratios $(20,50$, 80 , and $100 \%$ tumors). First of all, we evaluated the accuracy (ACC), sensitivity (SE), and specificity (SP) in determining whether to phase segments in Accurity and CNAHap (Figure 2a, Supplementary Table S1a). CNAHap shows a higher accuracy and sensitivity than Accurity. Figure $2 b$ and Supplementary Table S1b display that CNAHap has more correctly called allele-specific CNVs than Accurity. Figure $2 \mathrm{c}$ is the histogram plot of phased block length in CNAHap. Despite the purity, the majority (all $>93.88 \%$ ) of phased CNV segments' block length is larger than 100kbp (Supplementary Table S1c). As illustrates in Figure 2d and Supplementary Table S1d, CNAHap achieves high SNVs phase rates, all larger than $99.98 \%$ regardless of the purities. The number of phased SNVs in purity 1 for samples sim_1, sim_2, and sim_3 is 33740, 32477, and 34436, respectively. With the decrease of the tumor purity, the number of phased SNVs increased. The reason might be that the increase of normal reads in synthetic mixtures adds bias on the CNV segmentation procedure, yielding longer $\mathrm{CNV}$ segments qualified for phase. The statistical difference of purity $0.2-0.5$ vs. purity 1 is much higher than purity 0.8 vs. 1. In Figure 2e$\mathrm{f}$ and Supplementary Table S1e, we observe that the mean of switch error and mismatch error in purity 1 samples are 0.0270 (SD: 0.0567, Median: 0, IQR: 0-0.0153) and 0.0294 (SD: 0.0471, Median: 0, IQR: 0-0.0441). The switch error and mismatch error on samples with purity 0.2 and 0.5 are significantly higher than purity 1 samples (p-value of SE: 6.7e-16 and 5.8e-05, p-value of mismatch error: $<2.22 \mathrm{e}-16$ and $1.2 \mathrm{e}-05$ ). In contrast, samples between purity 0.8 and 1 tell no significant difference in error rate. To summarise, our synthetic experiments reveal that as long as the tumor purity larger than 0.5, CNAHap enables producing trustable copy numbers and phase profiles.

\section{Case study on a Hepatocellular carcinoma cohort.} Hepatocellular carcinoma (HCC) is one of the leading causes of cancer death (24). Sung et al. have studied hepatitis B virus (HBV) integration in liver cancer genomes by leveraging the whole-genome sequencing of HCC tumors and adjacent normal tissues (25). In the present case study, we applied CNAHap to reanalyzed the data focusing on putative cancerrelated gene amplification with phased haplotypes.

As experimented in in silico datasets, CNAHap is sensitive to tumor purity. Thus, we filtered out all samples smaller than or equal to 0.5 (Figure 3a). Then, we selected tumors with prevalent large copy number abberations across the genome (Figure 3b). As a result, 24 HCC samples remained. The circos plot Figure $3 \mathrm{c}$ demonstrates the 24 HCC samples are prevalent with copy number gains and allele imbalance across the genome. CNAHap also deciphered the major and minor copy number of each CNV segment. We run GISTIC2 (26), RAIG (22), and RUBIC (23) to check the focal $\mathrm{CNV}$ events. As illustrated in Figure 3d and Supplementary Table S2a, RUBIC detected one significant (q-value $<0.25$ ) 
a

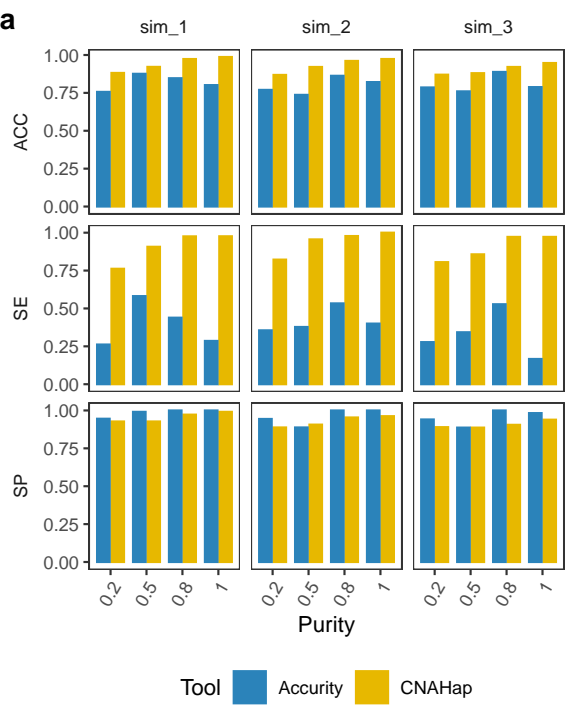

b

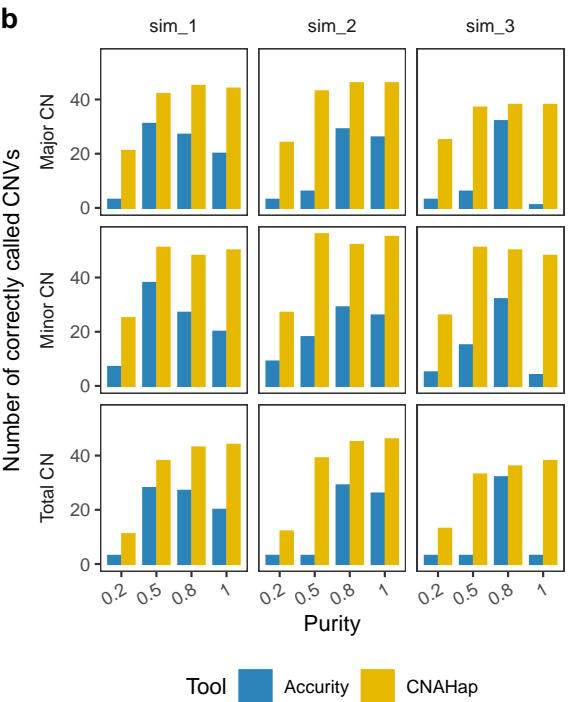

c
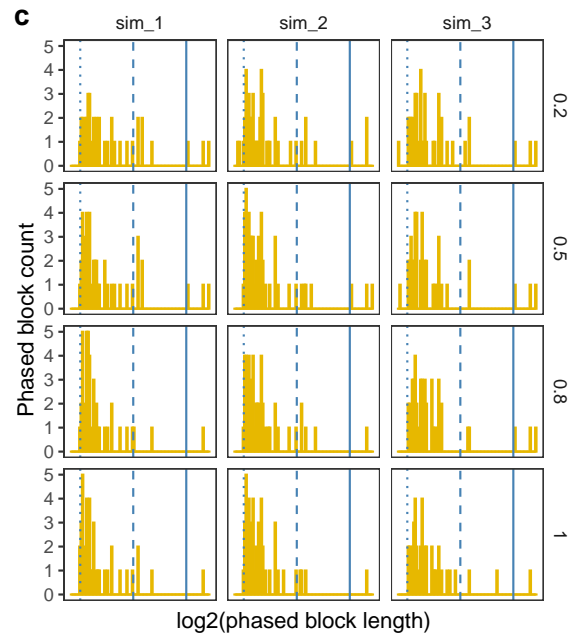

d

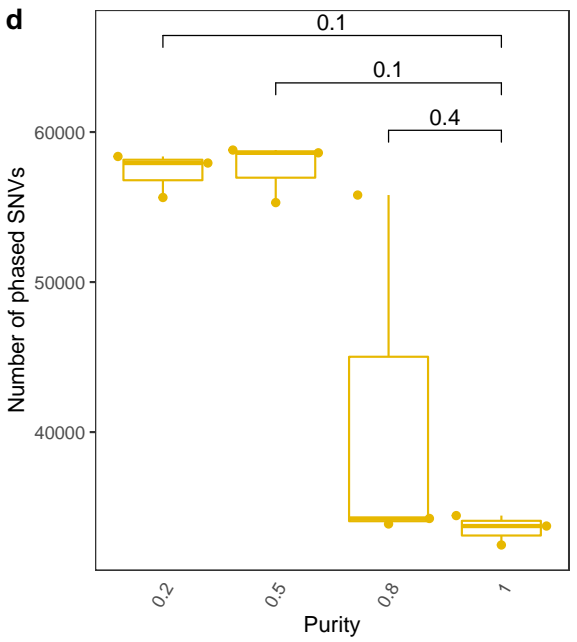

Tool CNAHap

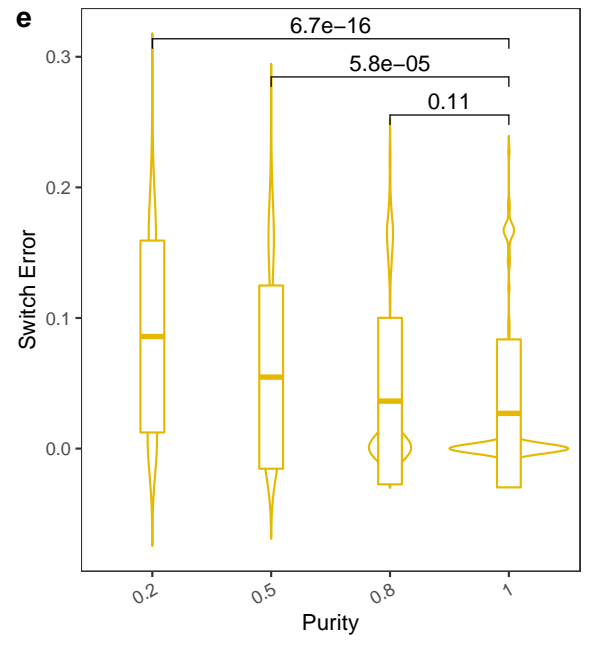

Tool $\square$ CNAHap \begin{tabular}{ll|l:ll} 
Position $\vdots 100 \mathrm{k}$ & $10 \mathrm{M}$ & $1 \mathrm{M} \quad$ Tool $\square$ CNAHap
\end{tabular}

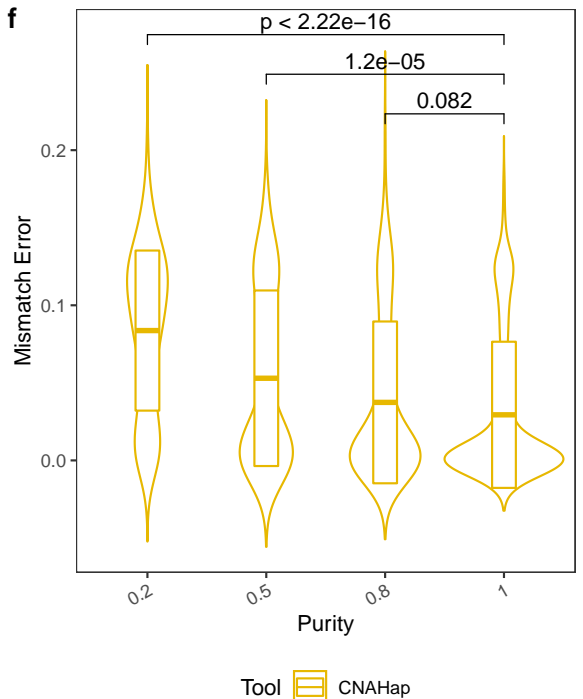

Fig. 2. Evaluation of synthetic data among different ploidy. (a) The accuracy (ACC), sensitivity (SE), and specificity (SP) in determining whether to phase CNV segments in Accurity and CNAHap. (b) The number of correctly called CNVs in Accurity and CNAHap. (c) The histogram plot of phased block length in CNAHap. (d) The number of phased SNVs in CNAHap. (e-f) The swith error and mismatch error in CNAHap.

amplification region chr20:25849750-30020750; RAIG $_{320}$ detected 10 significantly (q-value $<0.25$ ) amplified region: 321 chr1:15001-563000, chr4:14001-68500, chr5:1547501-з22 1920500, chr5:17635001-17922500, chr8:12046001- з2з 12315500, chr8:1923001-2332000, chr10:38769001- з24 38889000, chr12:1-148500, chr14:106785501-107289000, ${ }_{225}$ and chr14:19000001-19153000. We abandoned GISTIC2 as 326 it produced lots of focal deletions in contrast with the truth ${ }_{327}$ of no deleted segment was called among 24 HCC samples (Figure 3c). Among the 11 focal CNV events, 53 genes ${ }^{328}$ were annotated (Supplementary Table S2a), and their total, ${ }^{329}$ major, and minor copy number are depicted in the heatmap ${ }^{330}$ Figure $3 \mathrm{e}$. We found that 12 genes are previous reported to ${ }^{331}$ show focal CNV event in another Chinese HBV associated ${ }^{332}$ HCC cohort (27) (focal gains on DEFB109P1B, FAM138A, ${ }^{333}$ FAM138F, FAM66A, LOC100132062, LOC100132287, 334 LOC100133331, OR4F16, OR4F29, OR4F3, OR4F5; focal ${ }^{335}$ loss on FAM86B2). (Supplementary Table S1b). Figure $3 \mathrm{f}^{336}$ demonstrates that focal amplified genes were significantly enriched (p-value $<0.05$ ) in 18 GO pathway and 1 KEGG pathway. Olfactory transaction pathway/olfactory receptor activity (focal gains on OR4F16, OR4F3, OR4F29, OR4F5, $O R 11 H 12$ ) are recognized as putative drivers of cancer (28). NADH dehydrogenase activities were associated with HCC (29). Kaszak et al. reported that cadherin binding associated with HCC (30).

Figure 4 demonstrates the CNAHap phasing profiles among 24 HCC samples. In Figure 4a, we observe that the phased $\mathrm{CNV}$ segments were dominant across the whole genome with the mean of genomic region proportion 78.78 (SD: 13.01, Median: 81.29, IQR: 75.23-87.97)\% (Supplementary Table S3a-b). Despite the number of phased CNV segments (Mean: 377.17, SD: 282.34, Median: 288, IQR: 155-578.25) varying across samples, the ratio of phased CNV segments is on average at 49.56 (SD: 09.22, Median: 46.28, IQR: 47.43-55.63)\% (Figure 4b, Supplementary Table S3a-b). Fig- 
bioRxiv preprint doi: https://doi.org/10.1101/2021.03.27.437314; this version posted March 29, 2021. The copyright holder for this preprint (which was not certified by peer review) is the author/funder, who has granted bioRxiv a license to display the preprint in perpetuity. It is made available under aCC-BY-NC-ND 4.0 International license.
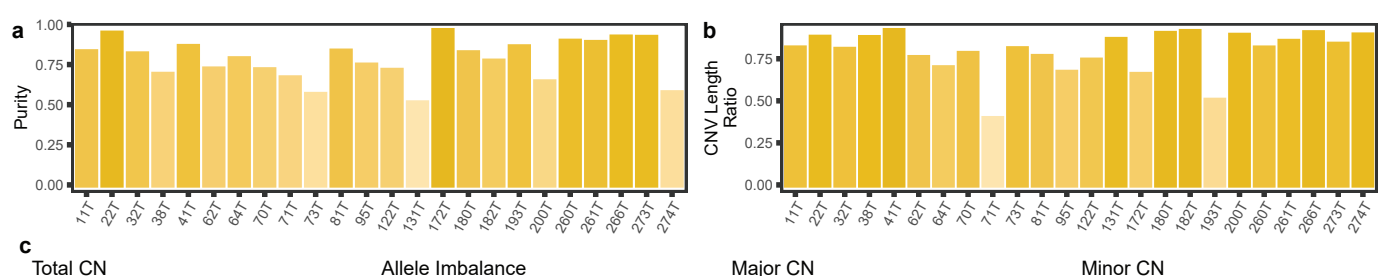

Total CN
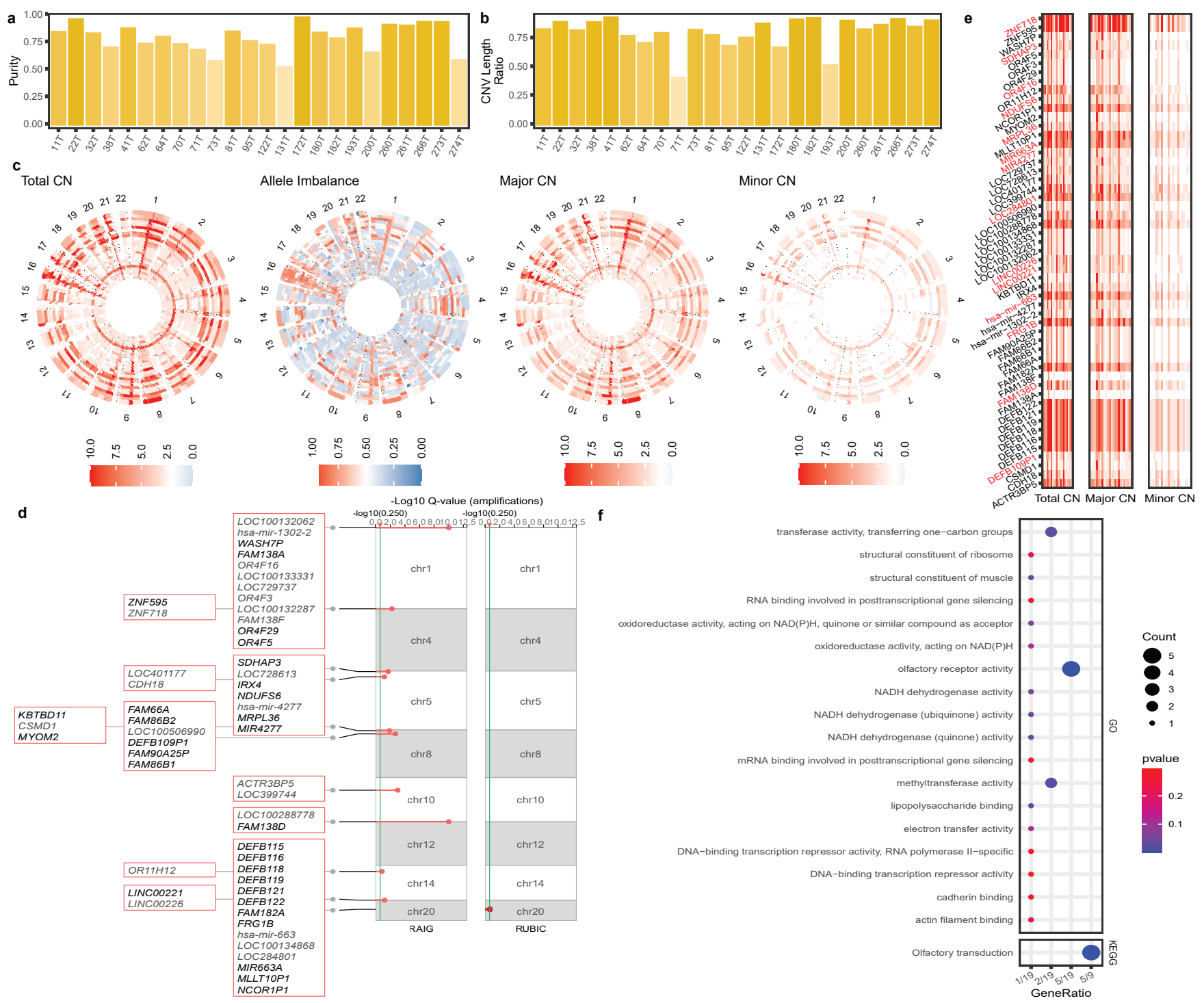

Fig. 3. HCC CNV profiles. (a) The estimated purity. (b) The CNV Segments region genomic proportion. (c) The circos plot of total CN, allele imbalance, major CN, minor CN across the genome, one circos layer represents one HCC sample. (d) The focal gains obtained from RAIG (22) and RUBIC (23). (e) The CNV heatmap of focal gain genes. (f) The enriched GO and KEGG pathway of focal gain genes. In (c) and (e), allele imbalance larger than, equal to, and less than 0.5 is annotated in red, white, and blue, respectively. Total $\mathrm{CN}$ larger than, equal to, and less than 2 is colored in red, white, and blue, respectively. Major/Minor $\mathrm{CN}$ larger than, equal to, and less than 1 is labeled in red, white, and blue, respectively. HCC: Hepatocellular carcinoma, CN: Copy Number.

ure 4c-d demonstrate that CNAHap generates large phasing ${ }_{355}$ blocks. The average N50 and N90 among HCC corhot is 356 around 25M and 7M, respectively. [N50 (Mean: 25,871,765, 357 SD: 19,222,539, Median: 21,537,499, IQR: 9,630,624 - 358 42,678,249) bp, N90 (Mean: 7,456,541, SD: 6,287,567, Me- 359 dian: 5,764,999, IQR: 2,701,124 - 10,852,249) bp]. The av- 360 erage number of phased SNVs is $1,763,850$ (SD: 323,620.1, ${ }_{361}$ Median: 1,839,513, IQR: 1,695,264-2,006,223) and the av- 362 erage phase rate is 78.83 (SD: 13.97, Median: 82.26 , IQR: ${ }_{363}$ $73.72-89.65) \%$. The long phasing block and high phasing ${ }_{364}$ rate are due to the phased CNV events occupying the ma- 365 jority of genome (Figure 4a), and around 93.77 (SD: 3.16, 366 Median: 93.98, IQR: 93.01-95.69)\% phased CNV segments ${ }_{367}$ are longer than 100k and around 66.78 (SD:10.50, Median: 368 66:31, IQR: 57.79-76.23)\% longer than 1M (Figure 4e, Sup- 369 plementary Figure S1a-b, Supplementary Table S3a-b), pro- 370 viding extreme long allele imbalance linkage to phase.
Then, we checked the phasing results of focal amplified genes. As demonstrated in Figure 4f, a total of 39 focal gain genes harbor SNV variants. Supplementary Figure S1c illustrates the scatter plot of focal gain gene mutation number and density, we can observe that CNAHap successfully phase genes with larger than 4,000 SNV variants. 23 out of 39 genes are completely phased in the cohort. Among them, LinkRNA LINC00221 are reported as a potential diagnostic and prognostic biomarker in HCC (31) (Figure 5a). FAM86B2 also shows focal CNV event in another Chinese HBV associated HCC cohort (27) (Supplementary Figure S2). 15 genes have less than four unphased samples, WASH7P has six unphased samples (Figure 5b). The mutation and phasing details for the rest of focal genes can be interactively visualized in web interface "Phased: On Genes" (https://bio.oviz.org/demo-project/ analyses/Phased_on_genes, Demo File: "CNA- 

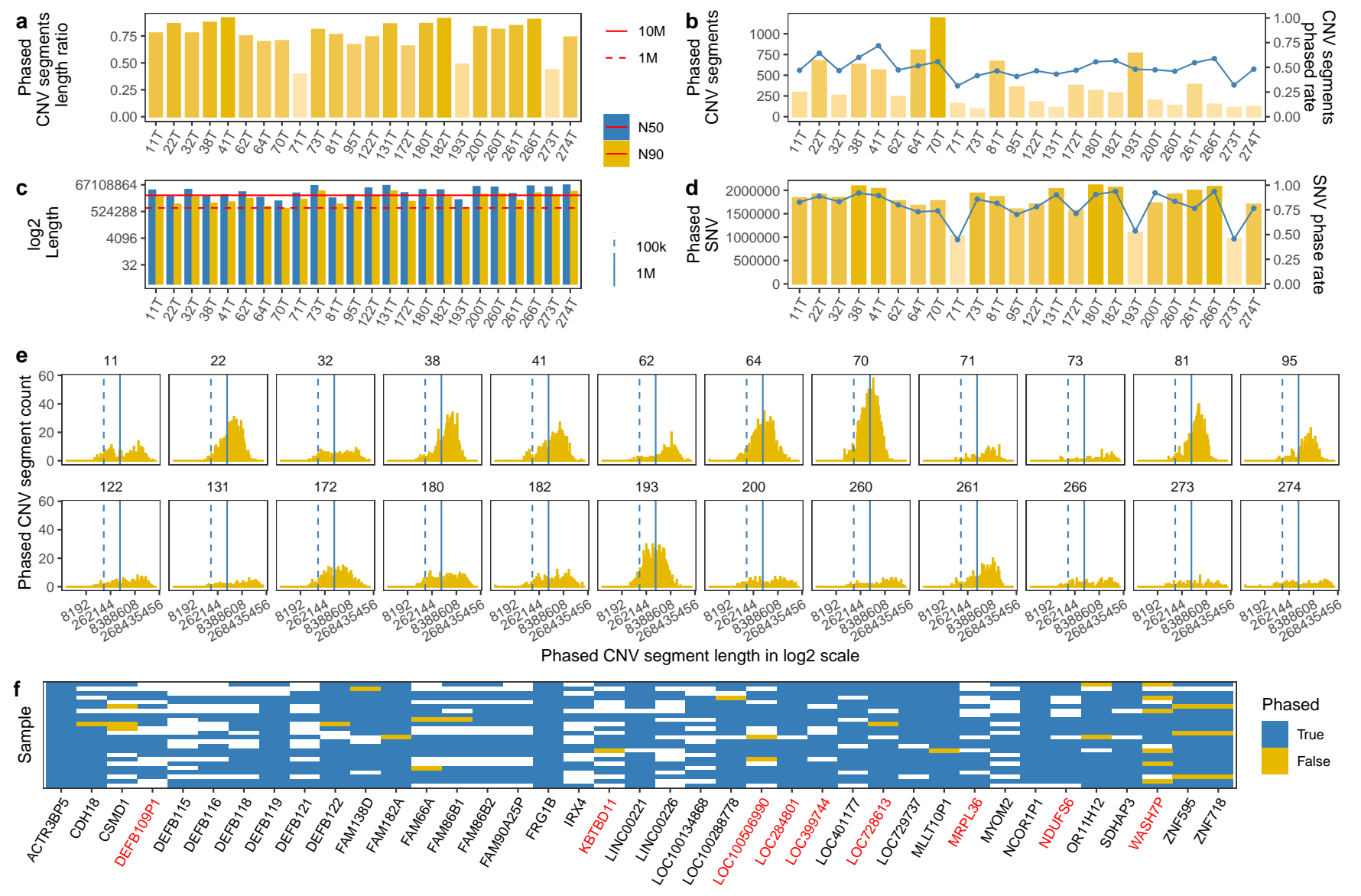

Fig. 4. HCC phased profiles among HCC samples. (a) The purity of tumor samples. (b) The number and proportion (blue line) of phased CNV segments. (c) The number and proportion (blue line) of phase SNVs. (d) The N50 and N90 of phased blocks, dashed and solid line indicates the length of 1M bp and 10M bp, respectively. (e) The histogram plot of phased CNV block length. Dashed and solid blue line shows the length of $100 \mathrm{k}$ bp and $1 \mathrm{M}$ bp, respectively. (f) Overview of phase result of focal gain gene, blue means phased gene, yellow otherwise. White tile indicates there is no SNVs in that gene. Genes colored in red are in enriched GO and KEGG pathway.

\section{Hap_HCC").}

CNAHap online visualization interfaces. The CNV pro- 398 files and phasing profiles in text format are nonintuitive for users to perceive the landscape and differences within a patient cohort. Thus, we developed three online web interfaces ${ }^{39}$ (CNV: Circos View, CNV: Focal Cluster, and Phased: on 400 Genes) to visualize the output of CNAHap. Table 1 sum- 401 marises the key features of the provided interfaces. CNV: 402 Circos View, demonstrated as Figure 3a, displays the cir- 403 cos plot of total $\mathrm{CN}$, major $\mathrm{CN}$, minor $\mathrm{CN}$, allele imbalance, 404 phased information, etc., across the patient cohort. CNV: 405 Focal Cluster, showed as Figure 3b, shows the recurrent 406 gains and losses detected by multiple tools, and supports En- 407 sembl (32) annotation. Phased: On Genes, illustrated as Fig- 408 ure 5 and Supplementary Figure S2, displays the mutation de- 409 tail and phasing profile on genes, supporting transcript (En- 410 sembl (32)) and protein (Pfam (33)) annotation. Generally, 411 we offer the users an editor to upload the CNVHap outputs 412 to the server and adjust the figure display settings. We pro- 413 vide interactive tooltips to show the essential information of 414 a sample, a CNV segment, an SNV variant, and so on, as- 415 sisting users in seizing potential findings quickly. With one- 416 button clicked, users can download high-quality figures for 417 share or paper publishing. For demonstration, we have up- 418 loaded the raw data of Figure 3a, Figure 3b, Figure 5, and Supplementary Figure S2 as demo data set "CNAHap_HCC" in the editor.

\section{Discussion}

Although the heterozygous allelic imbalance from tumor tissue is widely utilized to infer somatic copy number alterations (SCNAs) $(21,34,35)$. Collaborating tumor allelic imbalance to phase germline variants has not been broadly adopted. Prepemariy studies on VAF phasing and HATS have established that this data attribute to the assembly of the germline haplotype. However, running these tools requires arduous efforts as VAF phasing provides no accessible source code (18), and HATS necessitates a training process first (19). Thus, we introduce CNAHap, an easy-use tool that leverages imbalance in SNV or InDel alleles in copy number gains region to phase germline haplotype. Like haplotype assembly tools, CNAHap only demands sequencing data and can phase rare and de novo variants. Surpass the assembly-based ones, CNAHap is not constrained by the read length and insert size of particular sequencing protocols, thus yields much greater phasing blocks. CNAHap also calls the allele-specific copy number aberrations in tumor cells.

The allele-specific CNV profiles and phasing profiles in text 
bioRxiv preprint doi: https://doi.org/10.1101/2021.03.27.437314; this version posted March 29, 2021. The copyright holder for this preprint (which was not certified by peer review) is the author/funder, who has granted bioRxiv a license to display the preprint in perpetuity. It is made available under aCC-BY-NC-ND 4.0 International license.

a
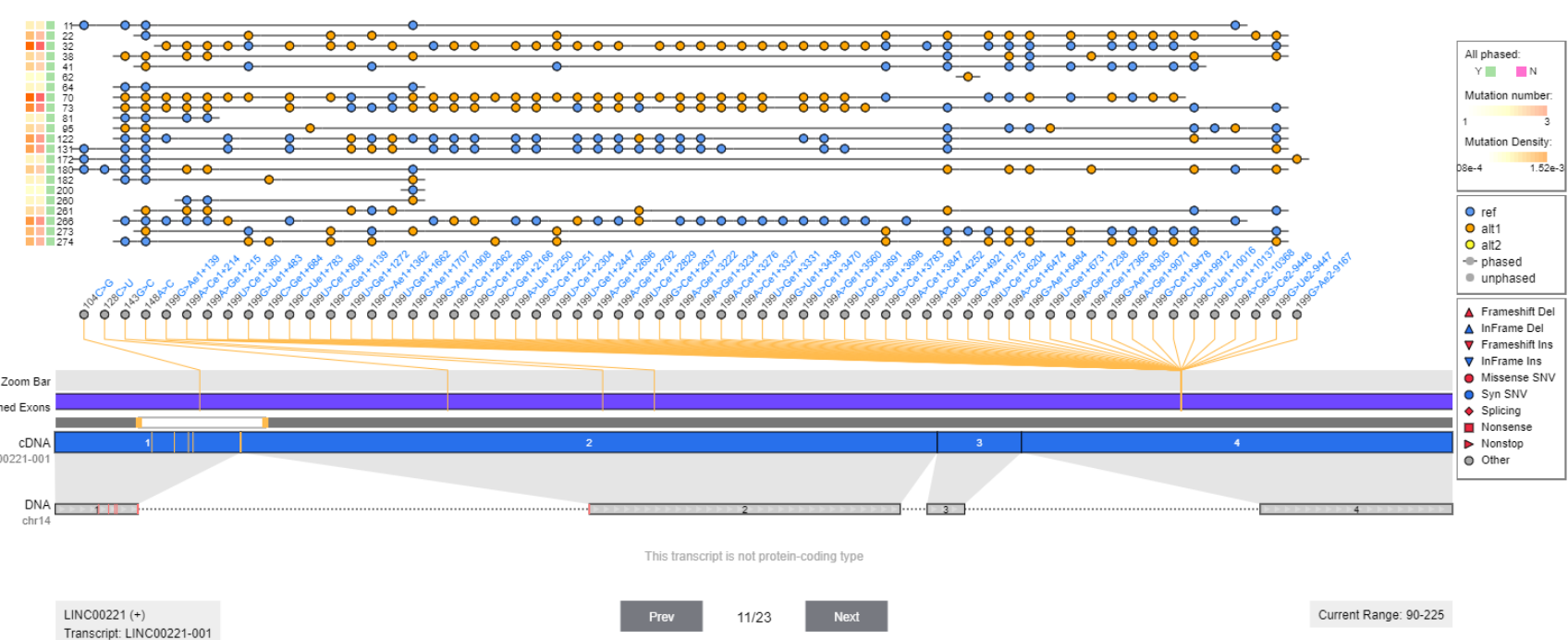

b

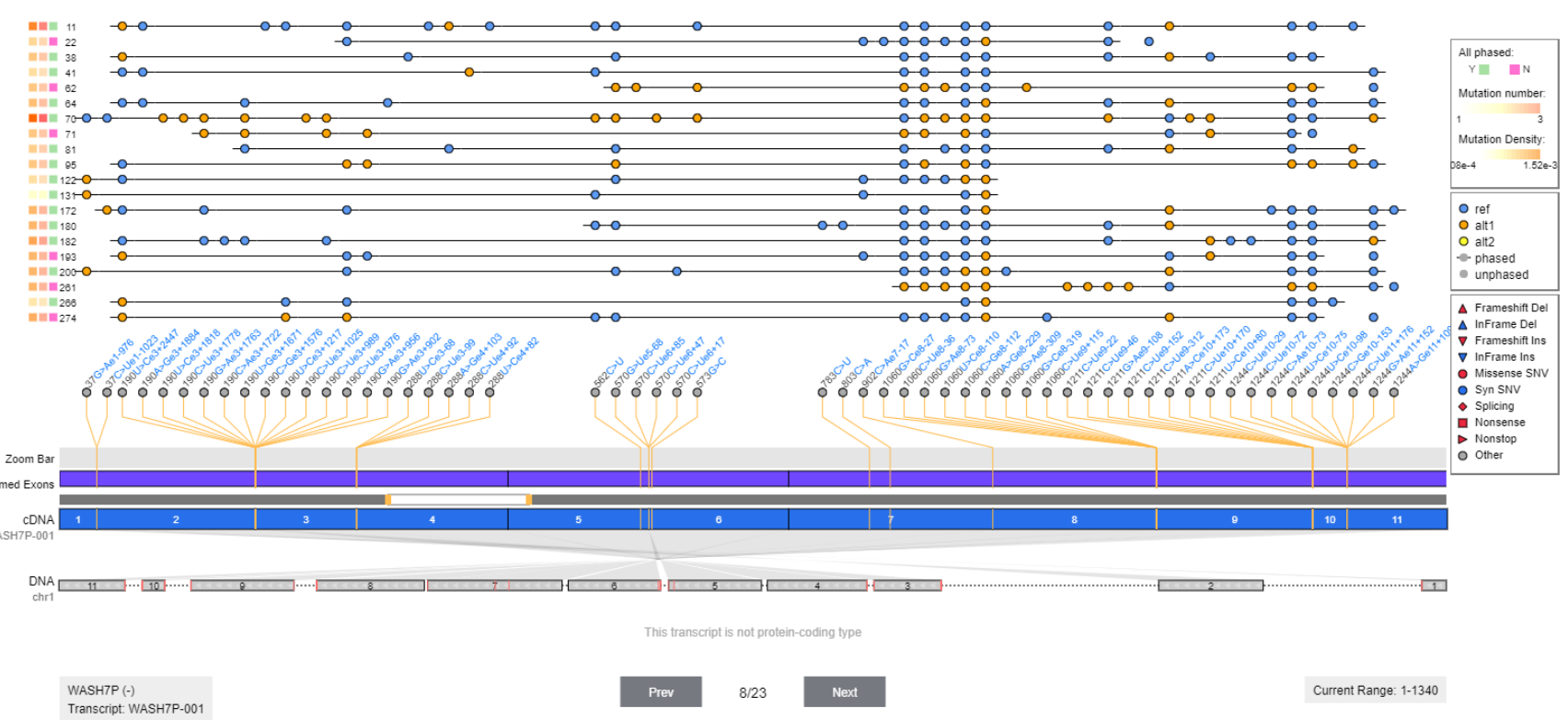

Fig. 5. (a-b) Phasing profile on lincRNA LINC00221 and gene WASH7P among the HCC cohort.

CNAHap visualization web interface

CNV: Circos View, e.g., Figure 3a

https://bio.oviz.org/demo-project/analyses/CNV_circos_view

CNV: Focal Cluster, e.g. Figure $3 b$

https://bio.oviz.org/demo-project/analyses/CNV_focal_cluster

Phased: On Genes, e.g. Figure 5, Supplementary Figure S2

https://bio.oviz.org/demo-project/analyses/Phased_on_genes

\section{Key Functionalities}

Circos plot of total, major, and minor copy number

Circos plot of allele imbalance

Circos plot of phased information

Recurrent gains and losses

Gene annotation Ensembl (32)

Illustrates multiple tools results parallelly

Phasing profile on genes, mutation detail information

Genes, transcripts annotation Ensembl (32)

Protein annotation Pfam (33)

Table 1. Summary of CNAHap visualization interfaces in bio. oviz . org Oviz-Bio (20).

format are nonintuitive for users to perceive the landscape 425 and differences within a patient cohort. To address this is- ${ }_{426}$ sue, we developed three online web interfaces (CNV: $\operatorname{Circos}_{427}$ View, CNV: Focal Cluster, and Phased: on Genes) to visual- ${ }_{428}$ ize the output of CNAHap. Equipped with interactive tooltips ${ }_{429}$ and editors, users can capture and share potential scientific ${ }_{430}$ discoveries without effort.

Noteworthily, some caveats need to be addressed. (1) CNAHap now only phases over the SCNA segments with allele imbalance but does not assign haplotype order in a balanced or diploid genomic region. In other words, CNAHap heavily pivots on the popularity and proportion of im- 
balanced somatic copy number alterations (SCNAs). Even ${ }_{483}$ though Compton et al. claimed that large CNV blocks are prevalent across the solid tumor genome (almost 90\%) (17), and our HCC case study reported the average SCNA pro- ${ }^{48}$ portion as 78.78 (SD: 13.01, Median: 81.29, IQR: 75.2387.97)\%. There exist near-diploid colorectal cancer (CRC) ${ }^{486}$ tumors (36), diploid lymph node metastases (37), diploid en- ${ }_{487}$ dometrioid adenocarcinomas (38), etc. Thus, we recommend ${ }_{488}$ fitting the paired normal data to assembly-based tool such ${ }_{489}$ as SpecHap (16) simultaneously and combining the phasing ${ }_{490}$ results between CNAHap and SpecHap to achieve a com- ${ }_{491}$ plete germline haplotype. (2) Our experiments on synthetic ${ }_{492}$ datasets indicate CNAHap is sensitive to tumor purity. The ${ }_{493}$ switch error and mismatch error on samples with purity $0.2_{494}$ and 0.5 are significantly higher than purity 1 samples (pvalue of SE: $6.7 \mathrm{e}-16$ and $5.8 \mathrm{e}-05$, p-value of mismatch error: $<2.22 \mathrm{e}-16$ and $1.2 \mathrm{e}-05)$. In contrast, samples between ${ }^{495}$ purities 0.8 and 1 tell no significant difference in error rate. 496 Thus, we advise practicing CNAHap only to tumors with pu- 497 rity larger than 50\%. (3) Currently, CNAHap requires SCNA segments as input. We suggest leveraging Patchwork (35) or ${ }_{498}$ Accurity (21) to identify SCNA segments first and then using CNAHap to refine the allelic specific copy numbers and the ${ }^{499}$ germline haplotypes in regions where demonstrate an imbalanced SNV/InDel allele. (4) In this study, we only validated ${ }_{500}$ the efficacy of CNAHap in pair-end sequencing reads. In fact, CNAHap can apply to any normal-tumor pairs notwith ${ }^{501}$ standing the sequencing technologies, as long as users have ${ }^{502}$ the CNV segmentation and point mutation VCF file of the ${ }^{503}$ normal-tumor pair. (5) CNAHap is unable to recognize the ${ }^{504}$ haplotype of somatic mutations. We are considering it as a future enhancement.

\section{Conclusion}

Haplotype phasing is significant in the study of human genetics. The pervasiveness of the large copy number variant ${ }_{514}^{513}$ segment in solid tumors brings possibilities to resolve long ${ }_{515}$ germline phasing blocks utilizing allele imbalance in tumor ${ }_{517}^{516}$ data. Although there are such studies, none of them provide 518 easy-use software on the premise of availability and usability. ${ }^{519}$ Herein, we present a novel method, CNAHap, to determine ${ }_{521}$ the copy number in tumor and then phase germline variants ${ }_{523}^{522}$ in tumor copy number segments with the aid of allele imbal- ${ }_{524}$ ance. We also provide interactive web interfaces to visualize ${ }_{526}^{525}$ the copy number and phase landscape of CNAHap. On in ${ }_{527}$ silico datasets, CNAHap demonstrates higher copy number ${ }_{529}^{528}$ calling accuracy than the benchmark tool and generates long ${ }_{530}$ phasing blocks. On a Hepatocellular carcinoma case study, ${ }_{532}^{531}$ CNAHap successfully generates huge phase blocks with the ${ }_{533}$ average N50 and N90 at 25M and 7M, respectively, and find ${ }_{535}^{534}$ the Olfactory receptor family is recurrent and amplified. In ${ }_{536}^{535}$ all, our results illustrate the efficacy of CNAHap in determin- ${ }^{537}$ ing tumor copy numbers and their long germline haplotypes. ${ }^{539}$

\section{Acknowledgements}

We would like to express sincere gratitude Mr. Yonghan Yu for his valuable assistance and advice.

\section{Contributions}

S.C.L. supervised the project. S.C.L., B.T., W.J. and L.C. discussed the algorithm. B.T. implemented the algorithm and evaluated the method in simulations. L.C. designed and performed the case study. L.C. and W.J. designed the visualization interfaces. Y.W. and H.L. implemented the visualization interfaces in Oviz-Bio. L.C. and B.T. wrote the manuscript. S.C.L. revised the manuscript. All authors read and approved the final manuscript.

\section{Funding}

This research is funded by the Hong Kong Innovation and Technology Fund (ITF 9440236).

\section{Conflict of interest statement.}

None declared.

\section{Data availability}

HCC data used in this paper can be retrieved under the accession ERP001196 (25). All experiments can be reproduced with the dedicated version of software with default arguments.

\section{Software availability}

CNAHap source code is deployed at https://github. com/bowentan/CNAHap.

\section{Reference}

1. Hongbo $\mathrm{Si}$, Haris Vikalo, and Sriram Vishwanath. Haplotype assembly: An information theoretic view. In 2014 IEEE Information Theory Workshop (ITW 2014), pages 182-186. IEEE, 2014.

2. Robert Ekblom and Jochen BW Wolf. A field guide to whole-genome sequencing, assembly and annotation. Evolutionary applications, 7(9):1026-1042, 2014.

3. Sharon R Browning and Brian L Browning. Haplotype phasing: existing methods and new developments. Nature Reviews Genetics, 12(10):703-714, 2011.

4. Conrad Donald F, Jakobsson Mattias, Coop Graham, Wen Xiaoquan, Wall Jeffrey D, Rosenberg Noah A, and Pritchard Jonathan K. A worldwide survey of haplotype variation and linkage disequilibrium in the human genome. Nature genetics, 38(11):1251, 2006.

5. Stacy L Musone, Kimberly E Taylor, Timothy T Lu, Joanne Nititham, Ricardo C Ferreira, Ward Ortmann, Nataliya Shifrin, Michelle A Petri, M llyas Kamboh, Susan Manzi, et al. Multiple polymorphisms in the tnfaip3 region are independently associated with systemic lupus erythematosus. Nature genetics, 40(9):1062, 2008.

6. 1000 Genomes Project Consortium et al. An integrated map of genetic variation from 1,092 human genomes. Nature, 491(7422):56, 2012.

7. International HapMap 3 Consortium et al. Integrating common and rare genetic variation in diverse human populations. Nature, 467(7311):52, 2010.

8. Ryan Tewhey, Vikas Bansal, Ali Torkamani, Eric J Topol, and Nicholas J Schork. The importance of phase information for human genomics. Nature Reviews Genetics, 12(3):215-223, 2011.

9. Gustavo Glusman, Hannah C Cox, and Jared C Roach. Whole-genome haplotyping approaches and genomic medicine. Genome medicine, 6(9):73, 2014.

10. Olivier Delaneau, Cedric Coulonges, and Jean-Francois Zagury. Shape-it: new rapid and accurate algorithm for haplotype inference. BMC bioinformatics, 9(1):540, 2008.

11. Vikas Bansal, Aaron L Halpern, Nelson Axelrod, and Vineet Bafna. An mcmc algorithm for haplotype assembly from whole-genome sequence data. Genome research, 18(8):13361346, 2008.

12. Siddarth Selvaraj, Jesse R Dixon, Vikas Bansal, and Bing Ren. Whole-genome haplotype reconstruction using proximity-ligation and shotgun sequencing. Nature biotechnology, 31(12):1111-1118, 2013. 
bioRxiv preprint doi: https://doi.org/10.1101/2021.03.27.437314; this version posted March 29, 2021. The copyright holder for this preprint

(which was not certified by peer review) is the author/funder, who has granted bioRxiv a license to display the preprint in perpetuity. It is made available under aCC-BY-NC-ND 4.0 International license.

13. Grace XY Zheng, Billy T Lau, Michael Schnall-Levin, Mirna Jarosz, John M Bell, Christopher M Hindson, Sofia Kyriazopoulou-Panagiotopoulou, Donald A Masquelier, Landon Merrill, Jessica M Terry, et al. Haplotyping germline and cancer genomes with high-throughput linked-read sequencing. Nature biotechnology, 34(3):303, 2016.

14. Peter Edge, Vineet Bafna, and Vikas Bansal. Hapcut2: robust and accurate haplotype assembly for diverse sequencing technologies. Genome research, 27(5):801-812, 2017.

15. Longzhi Tan, Dong Xing, Chi-Han Chang, Heng Li, and X Sunney Xie. Three-dimensional genome structures of single diploid human cells. Science, 361(6405):924-928, 2018.

16. Yonghan Yu, Lingxi Chen, Xinyao Miao, and Shuaicheng Li. Spechap: a diploid phasing algorithm based on spectral graph theory. BioRxiv, page 870972, 2021.

17. Duane A Compton. Mechanisms of aneuploidy. Current opinion in cell biology, 23(1):109113, 2011.

18. Alexandra R. Buckley, Trey Ideker, Hannah Carter, and Nicholas J. Schork. Rare variant phasing using paired tumor:normal sequence data. BMC Bioinformatics, 20(1):265-265, 2019.

19. Ninad Dewal, Yang Hu, Matthew L Freedman, Thomas LaFramboise, and Itsik Pe'er. Calling amplified haplotypes in next generation tumor sequence data. Genome research, 22(2):362-374, 2012.

20. Wenlong Jia, Hechen Li, Shiying Li, Lingxi Chen, and Shuai Cheng Li. Oviz-bio: a webbased platform for interactive cancer genomics data visualization. Nucleic Acids Research, 2020.

21. Z. Luo, X. Fan, Y. Su, and Y. S. Huang. Accurity: accurate tumor purity and ploidy inference from tumor-normal wgs data by jointly modelling somatic copy number alterations and heterozygous germline single-nucleotide-variants. Bioinformatics, 34(12):2004-2011, 2018.

22. Hsin-Ta Wu, Iman Hajirasouliha, and Benjamin J Raphael. Detecting independent and recurrent copy number aberrations using interval graphs. Bioinformatics, 30(12):i195-i203, 2014.

23. Ewald Van Dyk, Marlous Hoogstraat, Jelle Ten Hoeve, Marcel JT Reinders, and Lodewyk FA Wessels. Rubic identifies driver genes by detecting recurrent dna copy number breaks. Nature communications, 7(1):1-10, 2016.

24. Rebecca L Siegel, Kimberly D Miller, and Ahmedin Jemal. Cancer statistics, 2020. CA: a cancer journal for clinicians, 70(1):7-30, 2020.

25. Wing-Kin Sung, Hancheng Zheng, Shuyu Li, Ronghua Chen, Xiao Liu, Yingrui Li, Nikki P Lee, Wah H Lee, Pramila N Ariyaratne, Chandana Tennakoon, et al. Genome-wide survey of recurrent hbv integration in hepatocellular carcinoma. Nature genetics, 44(7):765-769, 2012.

26. Craig H Mermel, Steven E Schumacher, Barbara Hill, Matthew L Meyerson, Rameen Beroukhim, and Gad Getz. Gistic2. 0 facilitates sensitive and confident localization of the targets of focal somatic copy-number alteration in human cancers. Genome biology, 12(4):1-14, 2011.

27. Yanan Pang, Weixing Guo, Jiaqi Wang, Guixia Xu, Kai Cheng, Guangwen Cao, Mengchao $\mathrm{Wu}$, Shuqun Cheng, and Shanrong Liu. Gene copy number variations in the leukocyte genome of hepatocellular carcinoma patients with integrated hepatitis b virus dna. Oncotarget, 7(7):8006, 2016.

28. Marco Ranzani, Vivek Iyer, Ximena Ibarra-Soria, Martin Del Castillo Velasco-Herrera, Mathew Garnett, Darren Logan, and David J Adams. Revisiting olfactory receptors as putative drivers of cancer. Wellcome open research, 2, 2017.

29. Puo-Hsien Le, Shih-Chiang Huang, Siew-Na Lim, Chang-Hua Chou, Ta-Sen Yeh, TseChing Chen, Tzung-Hai Yen, Ming-Yao Su, Cheng-Tang Chiu, Chau-Ting Yeh, et al. Complex iv subunit 1 defect predicts postoperative survival in hepatocellular carcinoma. Oncology letters, 7(5):1430-1438, 2014.

30. Ilona Kaszak, Olga Witkowska-Piłaszewicz, Zuzanna Niewiadomska, Bożena DworeckaKaszak, Felix Ngosa Toka, and Piotr Jurka. Role of cadherins in cancer-a review. International Journal of Molecular Sciences, 21(20):7624, 2020.

31. Yanlin Feng, Souraka Tapara Dramani Maman, Xinyu Zhu, Xuefang Liu, Christian Cedric Bongolo, Chunzi Liang, and Jiancheng Tu. Clinical value and potential mechanisms of linc00221 in hepatocellular carcinoma based on integrated analysis. Epigenomics, (0), 2020.

32. Fiona Cunningham, Premanand Achuthan, Wasiu Akanni, James Allen, M Ridwan Amode, Irina M Armean, Ruth Bennett, Jyothish Bhai, Konstantinos Billis, Sanjay Boddu, et al. Ensembl 2019. Nucleic acids research, 47(D1):D745-D751, 2019.

33. Sara El-Gebali, Jaina Mistry, Alex Bateman, Sean R Eddy, Aurélien Luciani, Simon C Potter Matloob Qureshi, Lorna J Richardson, Gustavo A Salazar, Alfredo Smart, et al. The pfam protein families database in 2019. Nucleic acids research, 47(D1):D427-D432, 2019.

34. Peter Van Loo, Silje H Nordgard, Ole Christian Lingjærde, Hege G Russnes, Inga H Rye, Wei Sun, Victor J Weigman, Peter Marynen, Anders Zetterberg, Bjørn Naume, et al. Allelespecific copy number analysis of tumors. Proceedings of the National Academy of Sciences, 107(39):16910-16915, 2010.

35. Markus Mayrhofer, Sebastian DiLorenzo, and Anders Isaksson. Patchwork: allele-specific copy number analysis of whole-genome sequenced tumor tissue. Genome biology, 14(3):1$10,2013$.

36. Martine Muleris, Alexandra Chalastanis, Nicolas Meyer, Marick Lae, Bernard Dutrillaux, Xavier Sastre-Garau, Richard Hamelin, Jean-Francois Flejou, and Alex Duval. Chromosomal instability in near-diploid colorectal cancer: a link between numbers and structure. PLoS One, 3(2):e1632, 2008.

37. DAVID G. BOSTWICK and ISABELLE MEIERS. Chapter 32 - prostate. In Noel Weidner, Richard J. Cote, Saul Suster, and Lawrence M. Weiss, editors, Modern Surgical Pathology (Second Edition), pages 1121-1180. W.B. Saunders, Philadelphia, second edition edition, 2009.

38. Xavier Matias-Guiu. Chapter 7 - endometrial neoplasia. In Marisa R Nucci, Esther Oliva, and John R Goldblum, editors, Gynecologic Pathology, pages 233-259. Churchill Livingstone, Edinburgh, 2009 
bioRxiv preprint doi: https://doi.org/10.1101/2021.03.27.437314; this version posted March 29, 2021. The copyright holder for this preprint (which was not certified by peer review) is the author/funder, who has granted bioRxiv a license to display the preprint in perpetuity. It is made available under aCC-BY-NC-ND 4.0 International license.

\section{Supplementary Note 1: Supplementary Figures}
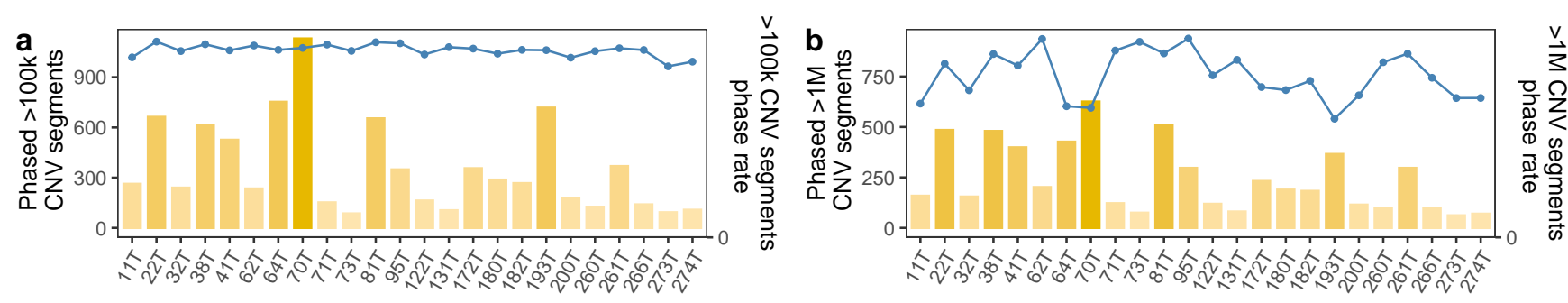

C
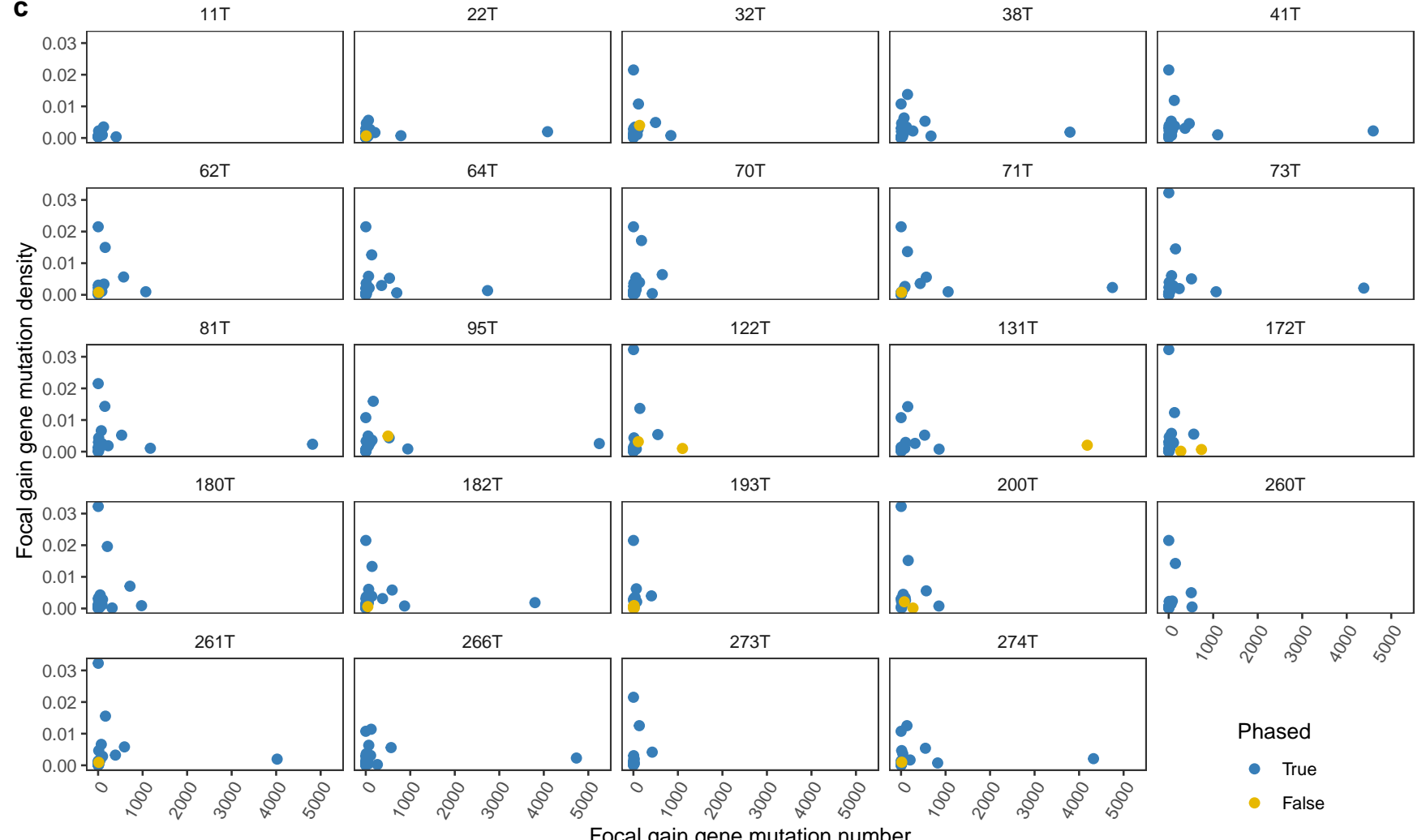

Phased

- True

Focal gain gene mutation number

Fig. S1. HCC phased profiles among HCC samples. (a) The purity of tumor samples. (b) The number and proportion (blue line) of phased CNV segments. (c) The number and proportion (blue line) of phase SNVs. (d) The N50 and N90 of phased blocks, dashed and solid line indicates the length of 1M bp and 10M bp, respectively. (e) The histogram plot of phased CNV block length. Dashed and solid blue line shows the length of $100 \mathrm{k}$ bp and $1 \mathrm{M} \mathrm{bp}$, respectively. (c) The scatter plot of focal gain gene mutation number and density, blue means phased gene, yellow otherwise. 
bioRxiv preprint doi: https://doi org/10.1101/2021.03.27.437314; this version posted March 29,2021 . The copyright holder for this preprint (which was not certified by peer review) is the author/funder, who has granted bioRxiv a license to display the preprint in perpetuity. It is made available under aCC-BY-NC-ND 4.0 International license.

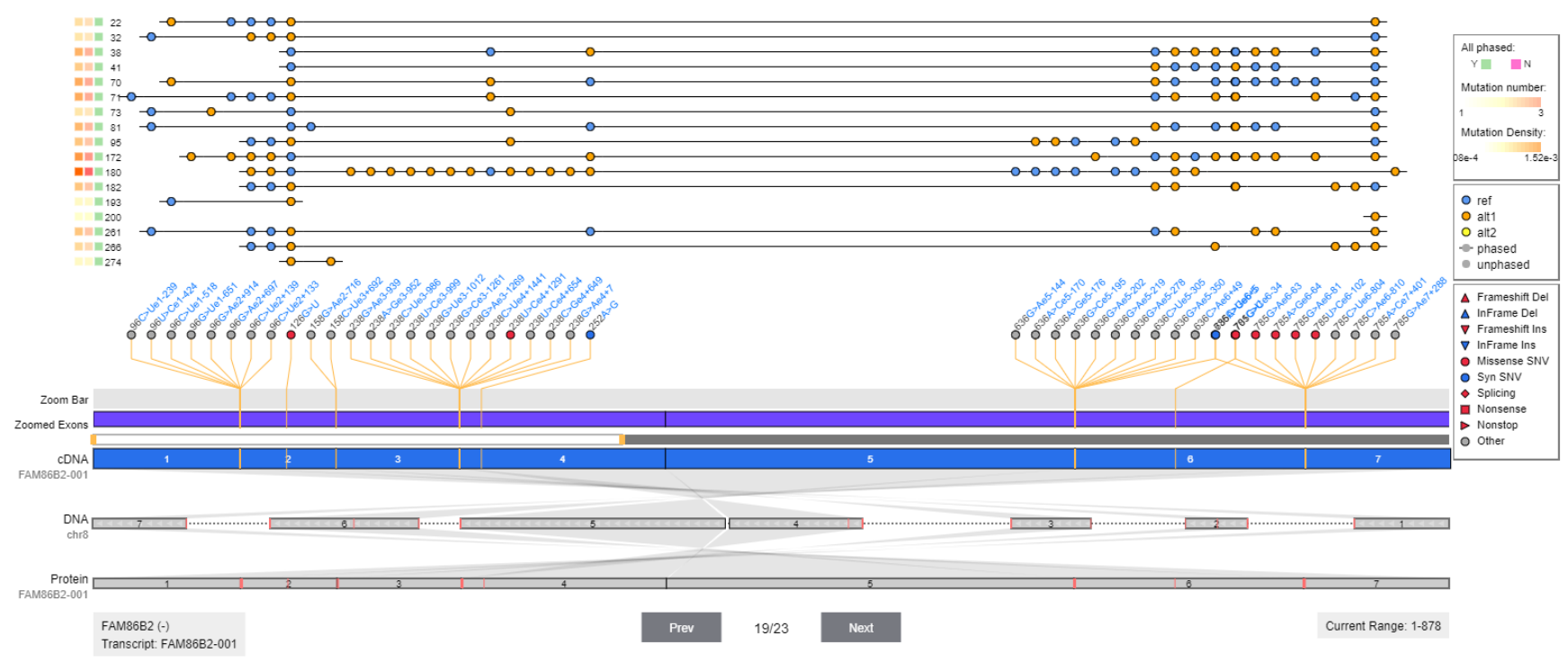

Fig. S2. Phasing profile on gene FAM86B2 among the HCC cohort. 

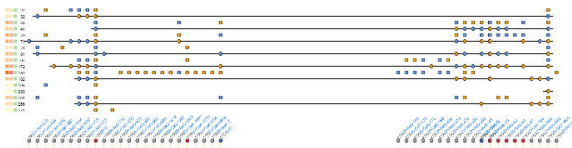

2... is 18

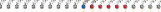
$\sin 90$

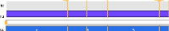

1

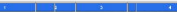

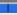
5. क $\square$ 


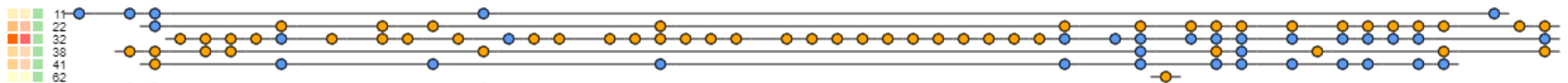

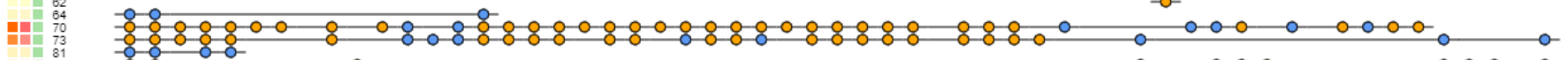

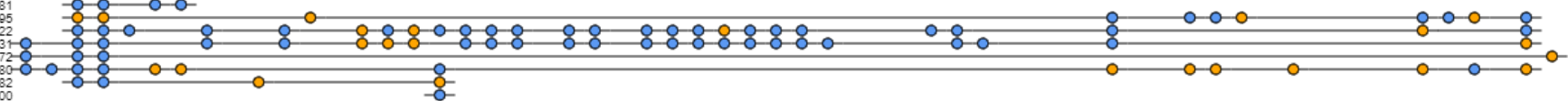

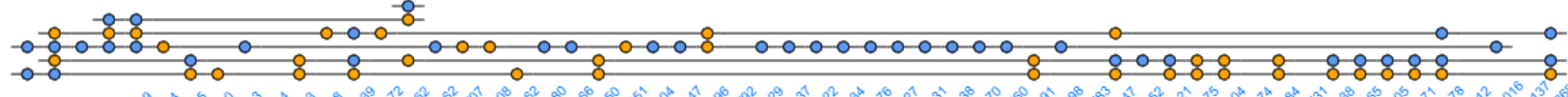

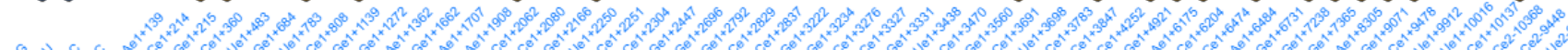

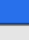

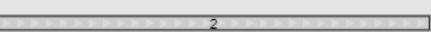

This transcript is not protein-coding type

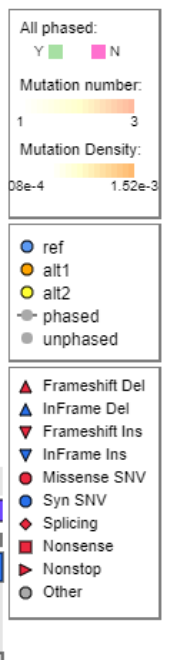

$11 / 23$

Next

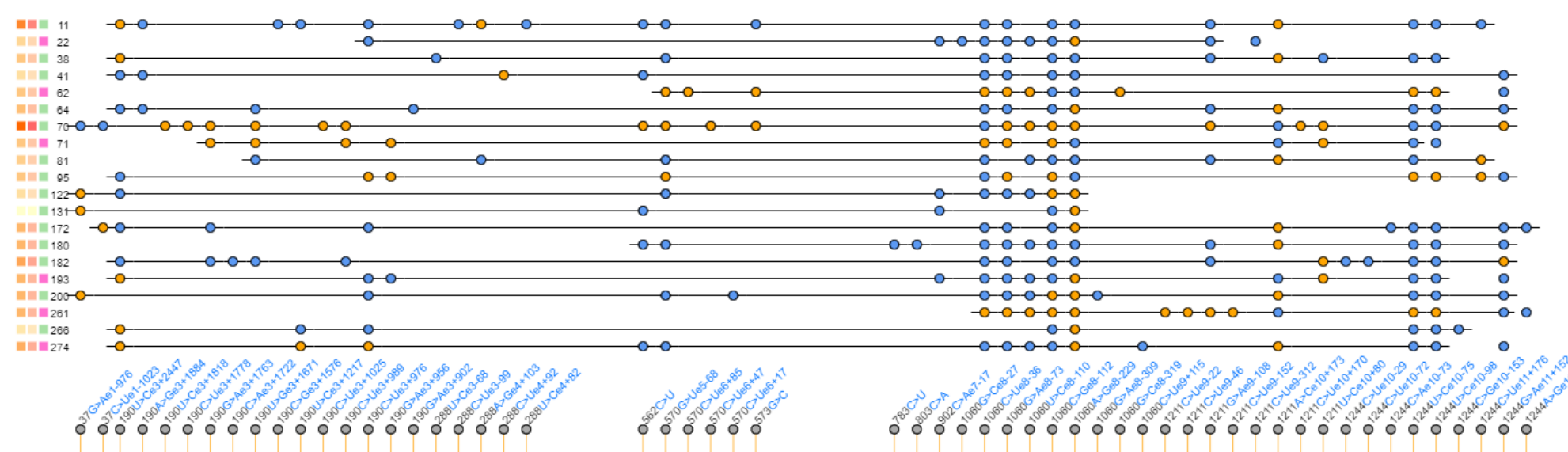

Zoom Bar

CDNA

000000

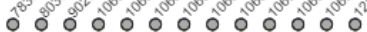

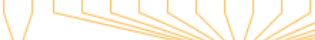

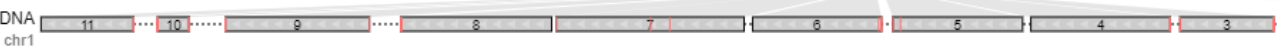




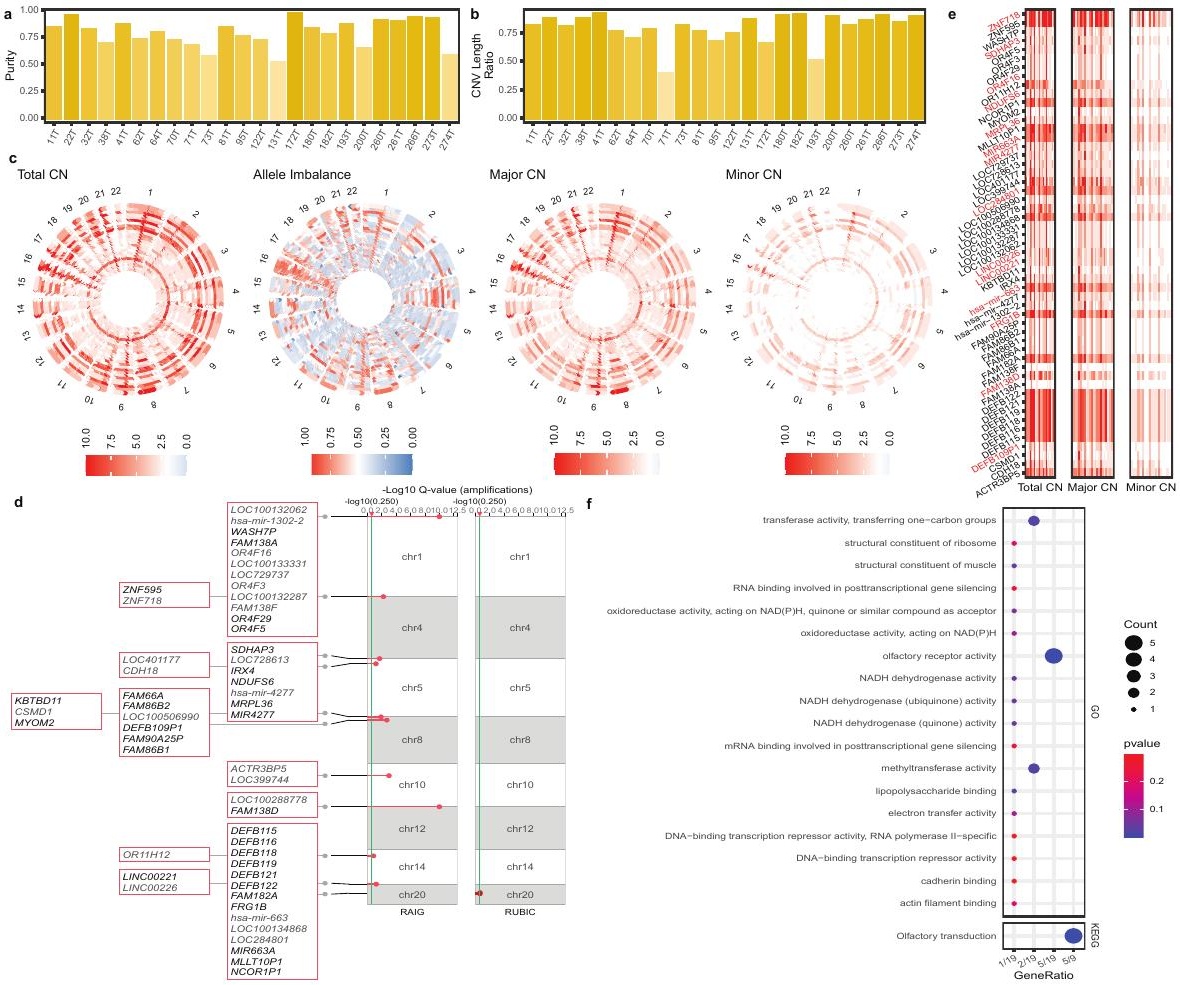



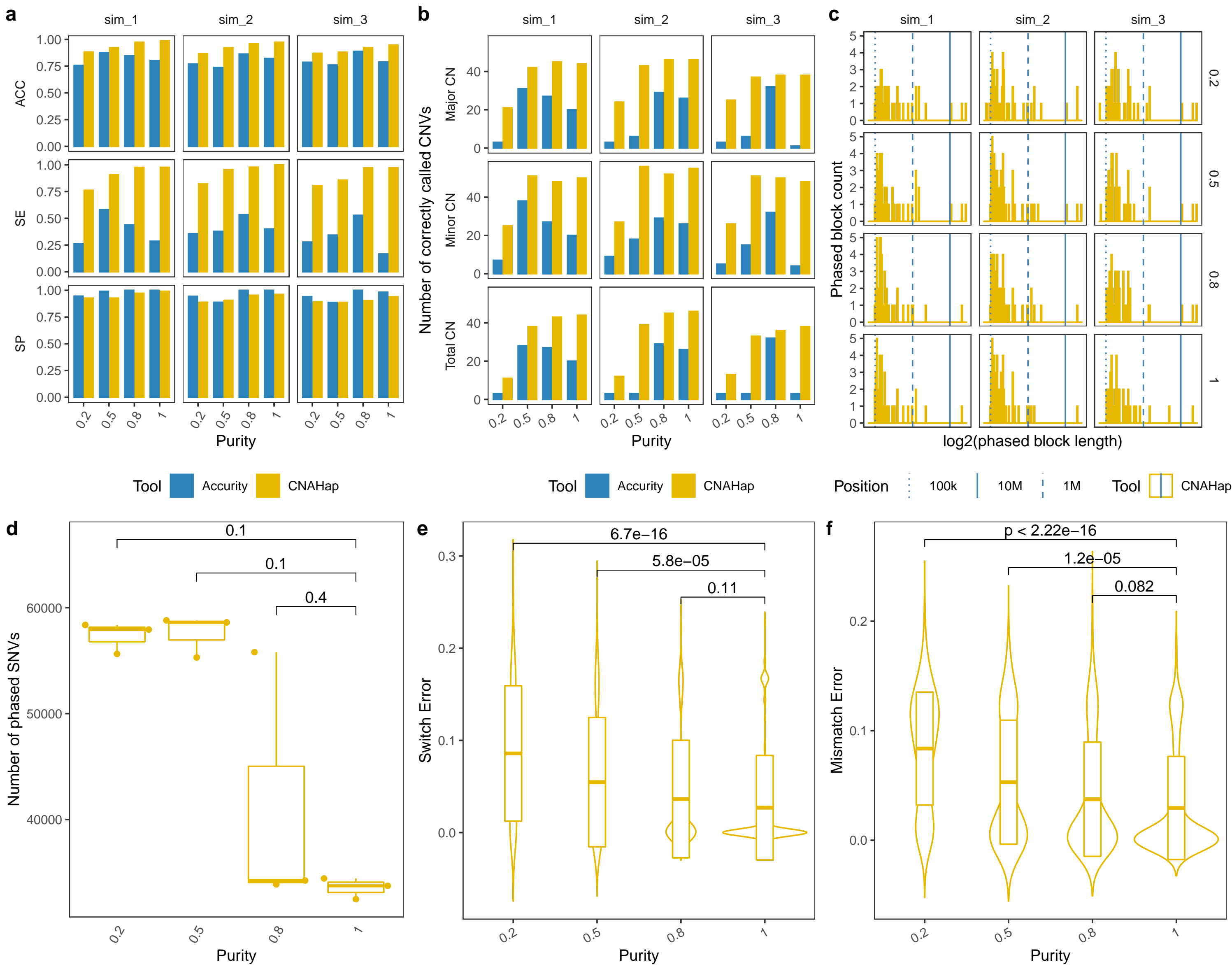

Tool CNAHap

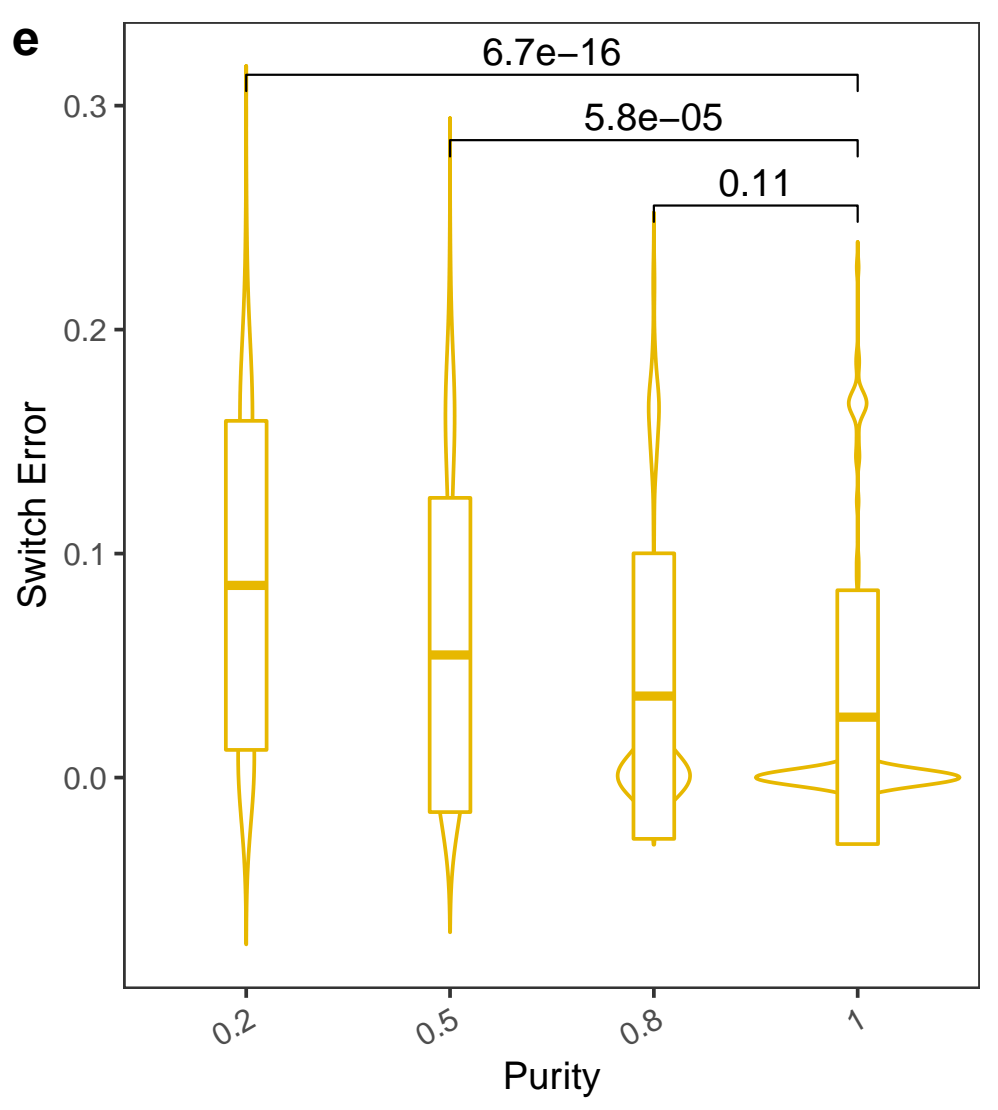

Position $\vdots 100 \mathrm{k} \mid 10 \mathrm{M} \quad 1 \mathrm{M} \quad$ Tool $\square$ CNAHap

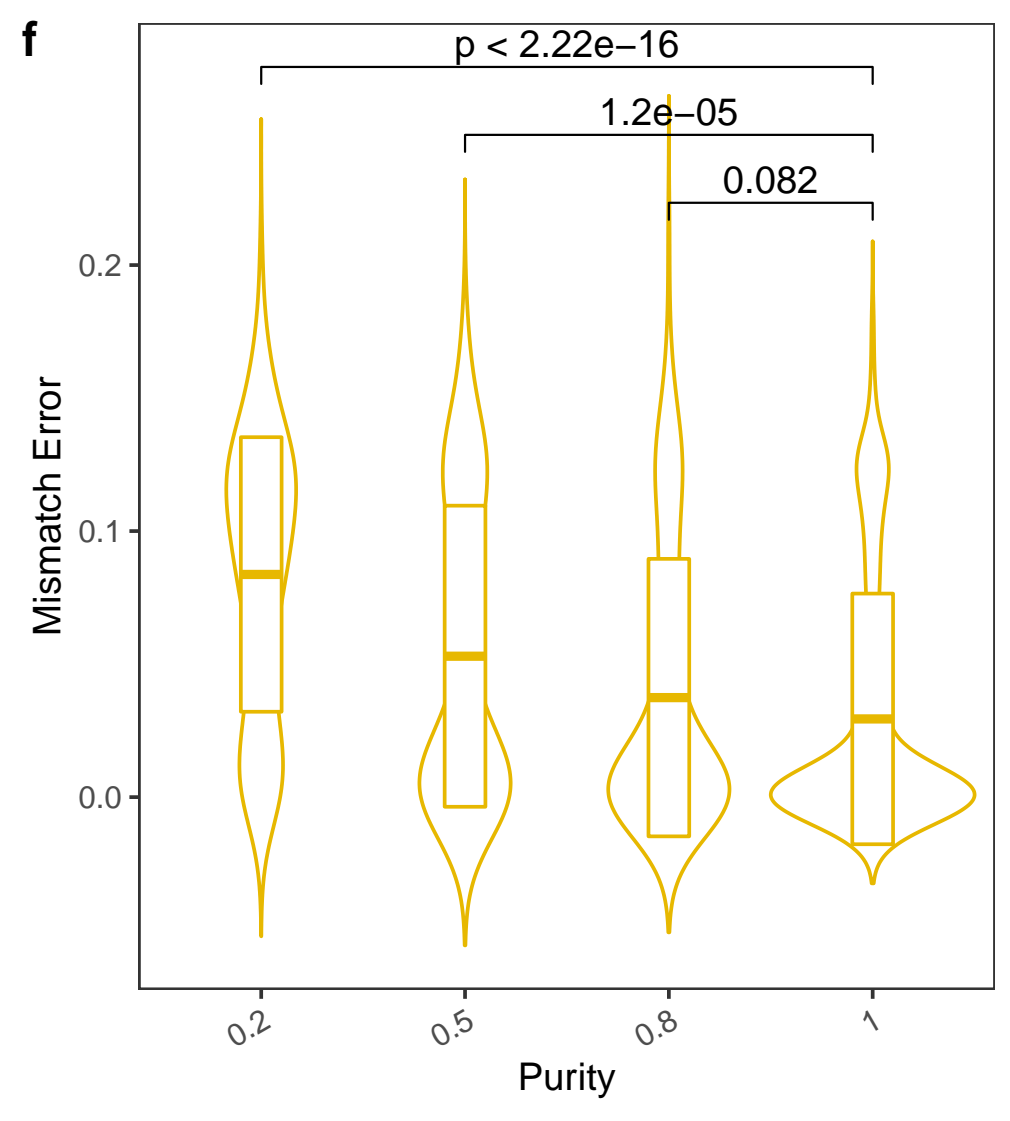

Tool $\square$ CNAHap

Tool $\square$ CNAHap 
a

Germline SNV
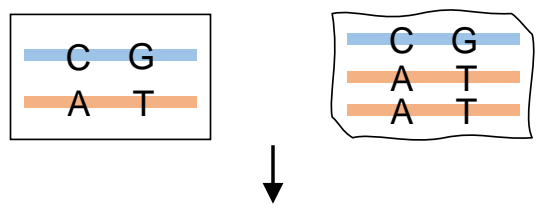

Sequencing reads
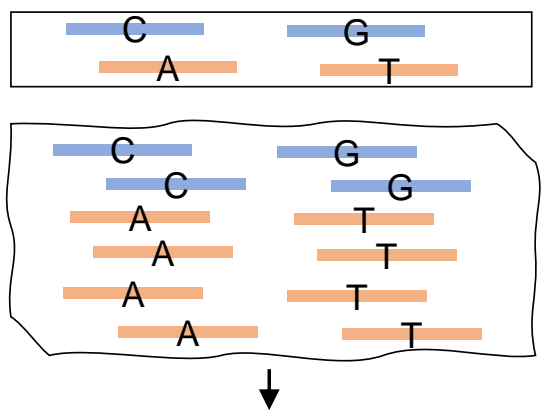

Phase with Allele Imbalance

Major Allele: AT

Minor Allele: CG

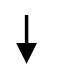

Resolved haplotypes

$$
\begin{array}{ll}
\hline C & G \\
A & T \\
A & T \\
\hline
\end{array}
$$

b

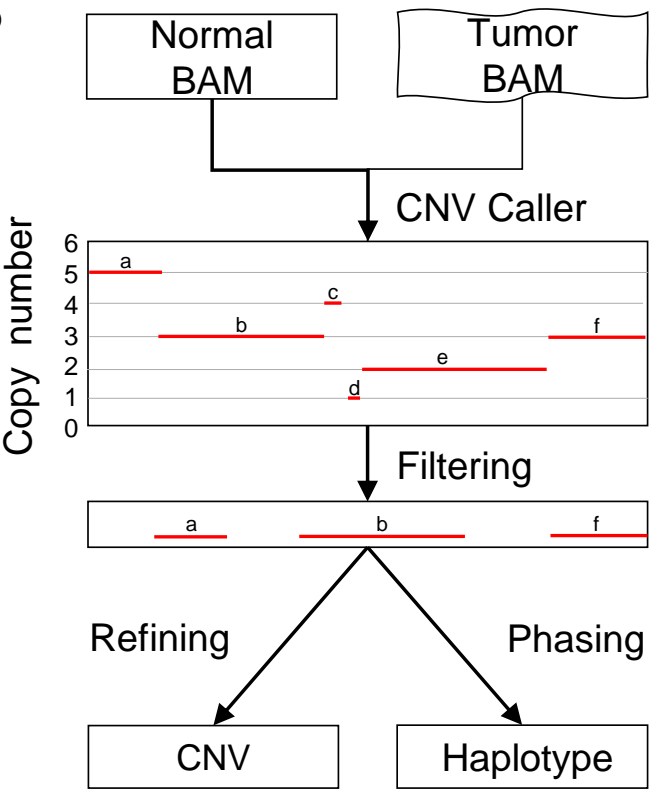

Filtering:

Filter out CNV segment which

- contains little SNVs "c" "d"

- not allele imbalanced "e"

Paternal $\quad \square$ Normal
Maternal $\quad \square$ Tumor

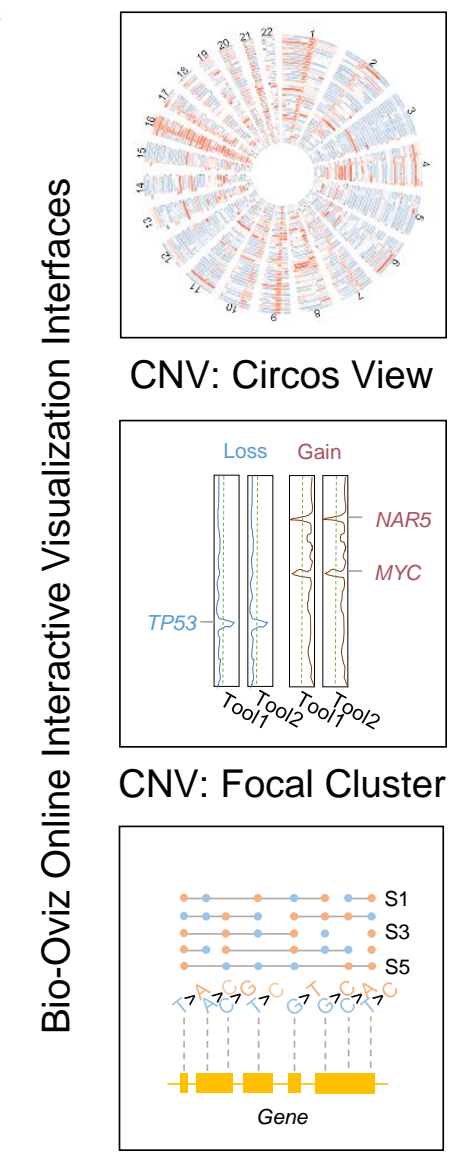

Phased: On Genes 